\title{
Ionic end-capping of (semi)telechelic polymers by mesogens: a novel route to liquid crystalline polymers
}

Gohy J-F [1], Jérôme R [1]

[1]Center for Education and Research on Macromolecules (CERM), Institute of Chemistry, B6, University of Liège, Sart-Tilman, B-4000 Liège, Belgium

\begin{abstract}
A mesogenic cation has been associated with low molecular weight carboxylato- and sulfonato(semi)telechelic polymers to form liquid crystalline halato(semi)telechelic polymers (LC H(S)TPs). Two methods have been used to end-cap the linear polymer chains at one (or both) end(s) by a mesogen through ionic bonding. The first method relies on the ion-exchange reaction between the metal counterion of halato(semi)telechelic polymers and an ionic mesogen. The second method is based on the proton-transfer from a sulfonic or carboxylic acid end-group to a tertiary aliphatic amine, this approach being controlled by the relative pKa's of the acid and basic groups.

The resulting materials have been characterized by differential scanning calorimetry (DSC), polarized optical microscopy (POM), small-angle X-ray scattering (SAXS) and small-angle neutron scattering (SANS).

On the basis of these experimental results, a model for the supramolecular organization of the LC $\mathrm{H}(\mathrm{S}) \mathrm{TPs}$ has been proposed. The final morphology results from the interplay of two competitive effects: the dipolar interaction of the ion-pairs known for multiplets formation and the propensity of the mesogenic moiety to form mesophases. The outcome of this competition depends on the mobility of the mesogenic counterion, i.e. on the strength of the dipolar interactions, the mobility of the polymer backbone and the mesogen/polymer ratio, which is controlled by the polymer molecular weight. A rodlike organization of the multiplets and a stretching of the polymer chains in the very close vicinity of the mesogenic core has been found in sulfonate polystyrenes, in agreement with the Eisenberg, Hird and Moore model for ionomers. The thickness of this region of restricted mobility has been estimated to $1 \mathrm{~nm}$, which is the order of magnitude of the persistence length of polystyrene.

Finally, LC H(S)TPs have been tested as interfacial agents in polystyrene/liquid crystal dispersions. The dipolar interactions of the ion pairs are clearly favorable to the additive localization at the polymer/LC interface. The higher polarity of the ammonium sulfonate pairs compared to the parent ammonium carboxylate ion pairs accordingly accounts for a higher interfacial activity.
\end{abstract}

Keywords Telechelic; SAXS; Liquid crystals; Ionomers; Supramolecular chemistry; Polymer dispersed liquid crystals

\section{Contents}

1. Introduction

2. Molecular characteristic features of the samples

2.1. Mesogens

2.2. Halato(semi)telechelic polymers $(\mathrm{H})$

2.3. Liquid crystalline halato(semi)telechelic polymers $(\mathrm{LCH})$

3. Differential scanning calorimetry and polarized optical microscopy of LCH

4. Small-angle X-ray scattering and small-angle neutron scattering studies of LCH

5. Model for the supramolecular organization of $\mathrm{LCH}$

6. Multiplet organization and restriction of mobility in $\mathrm{LCH}$

7. Comparison of LCH prepared by either ion-exchange or proton-transfer reaction

7.1. Differential scanning calorimetry and polarized optical microscopy analysis .....

7.2. Fourier-transform infrared spectroscopy analysis

7.3. Small-angle X-ray scattering analysis

8. Use of LCH as interfacial agents in polymer dispersed liquid crystals

9. Conclusions

Acknowledgements

References 


\section{Introduction}

In the recent years, non-covalent interactions, i.e. hydrogen-bonding, ionic interactions, coordination complexes and donor-acceptor interactions, were successfully used for building-up liquid crystalline polymers (LCPs), that consist of low molar mass mesogens linked to mutually reactive polymers [1-3]. The molecular assemblies formed by these macromolecules retain much attention for their contribution to the concept of 'Supramolecular Chemistry' introduced by Lehn [4].

An important class of LCPs, i.e. hydrogen bonded LCPs is based on hydrogen bonding between carboxylic (or benzoic) acid and pyridine moieties. Depending on the constitutive components, various liquid crystalline phases are observed. In this respect, side-chain hydrogen bonded LCPs have to be distinguished from hydrogen bonded main-chain LCPs. Kato, Frechet and coworkers investigated a variety of side-chain hydrogen-bonded LCPs based on benzoic acid-pyridine interactions [5-10]. In these systems, acid-pyridine complexation is thermodynamically favored over acid dimerization. Although it is not a prerequisite, mesogenic constituents are frequently used in the synthesis of these LCPs. An example of non-mesogenic precursor may be found in mixtures of functional vinyl polymers and mutually reactive non-liquid crystalline rigid aromatic derivatives, with no flexible spacer (or only a very short one) between the constitutive components [11]. These hydrogen-bonded LCPs form nematic phases.

Hydrogen-bonded main-chain LCPs were prepared by self-assembly of extended-chain structures based on mutually interacting small molecules. An elegant example was reported by Lehn et al. who mixed chiral tartaric acid with 2,6-diaminopyridine or uracil [12]. Although the precursors show a complex polymorphism, the mixture displays a liquid crystalline mesophase, whose the superstructure strongly depends on the chirality of the tartaric acid. In the same line, Griffin and coworkers reported the synthesis of main-chain LCPs by hydrogen bond-driven association of bispyridyne-terminated molecules and bisbenzoic acid-terminated compounds [13,14].

Ionic LCPs are not very popular compared to hydrogen-bonded assemblies and are mostly based on side-chain type architecture. In this respect, thermotropic LCPs were prepared by mixing an ammonium cation containing liquid crystal with anionic polyelectrolytes [15-19]. In a recent paper, Bazuin and Tork showed that liquid crystallinity could be imparted to ionically bonded side-chain type assemblies based on non-LC components [19]. Although a poly electrolyte was used in all the previous examples, ionomer-based LCPs were also prepared. Ujiie and coworkers indeed copolymerized a mesogen containing methacrylate with a low molar amount of an ionic liquid crystal monomer with formation of a liquid crystalline ionomer [20].

This type of ionic LCPs may not be kept confusing with charged covalently bonded LCPs, such as quaternized poly(4-vinylpyridine) and quaternized poly(ethyleneimine) based LCPs previously studied by Gramain et al. [21-24]. Liquid crystalline ionomers were also synthesized from covalently bonded LCPs, e.g. by partial hydrolysis of pendent ester groups of the main-chain [25].

Main-chain ionic LCPs were synthesized by interfacial condensation of a sulfonate-containing diol, 4,4/-dihydroxy- $\alpha, \alpha^{\prime}$-dimethylbenzalanine and a 50/50 mixture of sebacoyl and dodecanedioyl dichlorides [26].

This paper aims at reviewing the synthesis and characterization of a novel family of ionic LCPs, namely liquid crystalline halato(semi)telechelic polymers (LCH) [27-30]. These compounds have been designed as model liquid crystalline ionomers, since they consist of linear chains of well-defined molecular weight (MW) and narrow MW distribution, end-capped by a mesogen at one (or both) end(s) through ionic bonding.

This review will be subdivided into seven parts. First, the molecular characteristic features of the LCH will be briefly described. The liquid crystalline properties analyzed by differential scanning calorimetry (DSC) and polarized optical microscopy (POM) will be discussed in a second part. The supramolecular organization will be characterized in the third part by small-angle X-ray scattering (SAXS) and smallangle neutron scattering (SANS). In the fourth part, two models will be proposed for the supramolecular organization of LCH. The fifth part will be devoted to a detailed study of the multiplet organization of LC $\omega$-sulfonatopolystyrenes. A second synthetic route to LCH based on the protontransfer from an acid on the polymer to a base on the mesogen will be explored in the sixth part. Finally, application of LCH as interfacial agents in polymer dispersed liquid crystals will be discussed.

\section{Molecular characteristic features of the samples}

\subsection{Mesogens}

The structure and properties of the four mesogens under consideration in this paper are shown in Table 1. The mesogens M1 and M2, previously studied by Ujiie and Iimura [20], show liquid crystalline 
behavior. Conversion of M1 to the ionic version to form M2 considerably increases the thermal stability range of the smectic A mesophase, which explains that the mesogen M2 will be extensively used.

Mesogens M1, M3 and M4 will be essentially considered in Section 7. Indeed, a proton-transfer is expected to occur from the mixing of M1 with a sulfonic or carboxylic acid (semi)telechelic polymer. However, in order to avoid any interaction of the two hydroxyl groups of M1 (see Table 1) with the polymer acid end-group(s), M3 and M4 for sake of completeness were synthesized, which are the nonhydroxyl analogs of M1 and M2. Quite surprisingly, M3 and M4 are not liquid crystals, indicating that the two hydroxyl groups in M1 and M2 have a key role in stabilizing mesophases, as reported elsewhere [31].

$$
\begin{aligned}
& \mathrm{O}_{2} \mathrm{~N}-\left\langle 1, \mathrm{~N}=\mathrm{N}-\left\langle-\mathrm{O}-\left(\mathrm{CH}_{2}\right)_{6}-\mathrm{X}\right.\right. \\
& \text { M1: X stands for } \mathrm{N}-\underset{\mathrm{CH}_{2} \mathrm{CH}_{2} \mathrm{OH}}{\mathrm{CH}_{2} \mathrm{CH}_{2} \mathrm{OH}} \\
& \text { M2: } \mathrm{X} \text { stands for } \stackrel{\mathrm{N}}{+} \underset{\mathrm{I}^{-}}{+} \mathrm{CH}_{2} \mathrm{CH}_{2} \mathrm{OH} \\
& \text { M3: X stands for } \mathrm{N} \smile \underset{\mathrm{CH}_{2} \mathrm{CH}_{3}}{-\mathrm{CH}_{2} \mathrm{CH}_{3}} \\
& \text { M4: X stands for }{\stackrel{\mathrm{I}}{\mathrm{N}}>\mathrm{CH}_{2} \mathrm{CH}_{3}}_{\mathrm{I}^{-}}^{\mathrm{CH}_{2} \mathrm{CH}_{3}}
\end{aligned}
$$

Table 1 Structure and properties of the mesogens

$\begin{array}{ll}\text { Sample } & \text { Phase transition temperatures }\left({ }^{\circ} \mathrm{C}\right) \\ \text { M1 } & \text { Crystal } 60 \text { Smectic A mesophase 120 Isotropic } \\ \text { M2 } & \text { Crystal 41 Smectic A mesophase 170 Isotropic } \\ \text { M3 } & \text { Crystal 155 Isotropic } \\ \text { M4 } & \text { Crystal 162 Isotropic }\end{array}$

\subsection{Halato(semi)telechelic polymers $(H)$}

Anionically synthesized low molecular weight $\omega$ - and $\alpha, \omega$-sulfonato- and carboxylato-polystyrenes, polyisoprenes and poly(tert-butylacrylates) were used as halato(semi)telechelic polymers [32-35]. Table 2 lists the molecular characteristics of the halato(semi)telechelic polymers used in this study. 2.3. Liquid crystalline halato(semi)telechelic polymers ( $\mathrm{LCH}$ )

$\mathrm{LCH}$ were prepared by either ion-exchange (LCH used in Sections 3-6 and 8) or acid-base reaction (Section 7).

Ion-exchange between ammonium iodide bearing molecule and $\mathrm{Na}$ or $\mathrm{Li}$ salts end-capped polymer is schematized below. The reaction equilibrium was displaced towards the formation of the LCH by repeated water extraction of the accordingly formed micro-ions:

$\mathrm{P}-\left(\mathrm{A}^{-} \mathrm{X}^{+}\right) 1$ or $2+\mathrm{M}^{+} \mathrm{I}^{-}<=>\mathrm{P}-\left(\mathrm{A}^{-} \mathrm{M}^{+}\right) 1$ or $2+\mathrm{X}^{+} \mathrm{I}^{-}$

$\mathrm{P}=$ Polystyrene, polyisoprene, poly(tert-butylacrylate); $\mathrm{A}^{-}=\mathrm{SO}_{3}^{-}, \mathrm{COO}^{-}$;

$\mathrm{X}^{+}=\mathrm{Li}^{+}, \mathrm{Na}^{+} ; \mathrm{M}^{+}=\mathrm{M} 2, \mathrm{M} 4$.

The acid end-capped polymers were also neutralized by M1 and M3, as shown in Section 7. 
Table 2 Characteristic features of the H precursors

\begin{tabular}{|c|c|c|c|c|}
\hline Type of H & Acronym & $\bar{M}_{n}$ & Polydisperisty & Functionality (\%) \\
\hline$\omega$-Sulfonato polystyrene & PSS $1 / 0.7$ & 700 & 1.1 & 90 \\
\hline$\omega$-Sulfonato polystyrene & PSS $1 / 1.0$ & 1000 & 1.05 & 97 \\
\hline$\omega$-Sulfonato polystyrene & PSS $1 / 3.0$ & 3000 & 1.1 & 93 \\
\hline$\omega$-Sulfonato polystyrene & PSS $1 / 5.5$ & 5500 & 1.1 & 100 \\
\hline$\omega$-Sulfonato polystyrene & PSS $1 / 7.0$ & 7000 & 1.11 & 95 \\
\hline$\omega$-Sulfonato polystyrene & PSS 1/8.0 & 8000 & 1.05 & 98 \\
\hline$\alpha, \omega$-Sulfonato polystyrene & $\mathrm{PSS} 2 / 5.5$ & 5500 & 1.1 & 97 \\
\hline$\alpha, \omega$-Sulfonato polystyrene & $\mathrm{PSS} 2 / 7.0$ & 7000 & 1.07 & 84 \\
\hline$\alpha, \omega$-Sulfonato polystyrene & $\mathrm{PSS} 2 / 10.0$ & 10,000 & 1.12 & 97 \\
\hline$\omega$-Carboxylato polystyrene & PSC1/1.8 & 1800 & 1.15 & 84 \\
\hline$\omega$-Carboxylato polystyrene & $\mathrm{PSC} 1 / 2.0$ & 2000 & 1.15 & 85 \\
\hline$\omega$-Carboxylato polystyrene & PSC $1 / 4.5$ & 4500 & 1.15 & 95 \\
\hline$\omega$-Carboxylato polystyrene & PSC $1 / 6.0$ & 6000 & 1.05 & 92 \\
\hline $\begin{array}{l}\alpha, \omega \text {-Carboxylato } \\
\text { polystyrene }\end{array}$ & $\mathrm{PSC} 2 / 5.7$ & 5700 & 1.1 & 95 \\
\hline $\begin{array}{l}\alpha, \omega \text {-Carboxylato } \\
\text { polystyrene }\end{array}$ & $\mathrm{PSC} 2 / 6.0$ & 6000 & 1.1 & 95 \\
\hline$\omega$-Sulfonato polyisoprene & PIS1/4.5 & 4500 & 1.15 & 95 \\
\hline$\alpha, \omega$-Sulfonato polyisoprene & PIS2/11.0 & 11,000 & 1.1 & 92 \\
\hline$\omega$-Carboxylato polyisoprene & $\mathrm{PIC} 1 / 4.5$ & 4500 & 1.08 & 90 \\
\hline $\begin{array}{l}\alpha, \omega \text {-Carboxylato } \\
\text { polyisoprene }\end{array}$ & $\mathrm{PIC} 2 / 8.9$ & 8900 & 1.05 & 94 \\
\hline $\begin{array}{l}\alpha, \omega \text {-Carboxylato poly(tert- } \\
\text { butylacrylate) }\end{array}$ & $\mathrm{BAC} 2 / 3.8$ & 3800 & 1.08 & 90 \\
\hline $\begin{array}{l}\alpha, \omega \text {-Carboxylato poly(tert- } \\
\text { butylacrylate) }\end{array}$ & $\mathrm{BAC} 2 / 23$ & 23,000 & 1.05 & 95 \\
\hline
\end{tabular}

\section{Differential scanning calorimetry and polarized optical microscopy of LCH}

The thermal properties of the M2 containing LCH were studied by DSC and POM (Table 3). $\omega$ - and $\alpha, \omega$-sulfonato polystyrenes (except for the lowest MW) do not form liquid crystalline mesophases. The thermograms of these samples only show one glass transition $\left(T_{g}\right)$, which is generally lower than $T_{g}$ of the $\mathrm{H}$ precursor.

This modification is consistent with a plasticizing effect of the ionic mesogen. Plasticization was previously reported for non-covalent side-chain LCPs of low mesogen-content [6]. In contrast, the DSC trace for the lower molar mass PSS1M2/1.0 sample shows an increase in $\mathrm{T}_{\mathrm{g}}$ from 60 to $74^{\circ} \mathrm{C}$. It thus appears that the effect of an ammonium liquid crystalline counterion on $T_{g}$ of a sulfonate-ended polystyrene critically depends on the polystyrene molecular weight, i.e. on the mesogen-content. At low content, the ionic mesogen acts as a plasticizer, although an anti-plasticization is observed at higher mesogen contents.

A typical DSC curve for a $\omega$-sulfonato polystyrene is shown in Fig. 1. The detrimental effect of the ammonium sulfonates on the liquid crystalline properties is thought to result from the strong dipolar interactions of the ammonium sulfonates, that restrict the mobility of the mesogenic cation and thus its ability to form mesophase. This issue will be discussed in more details in Section 5.

Sulfonate LCH were observed by optical microscopy under crossed polarizers. Chloroform-cast films are homogeneous, transparent, red-colored and glassy at room temperature. They are not birefringent, as confirmed by POM. When the samples are heated above $T_{g}$ between two glass plates, the texture observed by POM consists of a dark region, in which there is no homeotropic alignment since no texture appears upon shearing. 
Table 3 Thermal properties of the $\mathrm{LCH}$ and the H precursors

Acronym Phase transition temperatures ${ }^{\mathrm{a}}\left({ }^{\circ} \mathrm{C}\right)$

$T_{\mathrm{g}}$ of the $\mathrm{H}$ precursors

$\left({ }^{\circ} \mathrm{C}\right)$

PSS1M2/0.7 GB 49.5 $5_{(4.9)}\left(T_{\mathrm{m}}\right)$ SA $155_{(1.85)}\left(T_{\mathrm{c}}\right) \mathrm{I}$

19

PSS1M2/1.0 G $\left(T_{\mathrm{g}}=74\right)$

PSS1M2/3.0 G $\left(T_{\mathrm{g}}=78\right)$

PSS1M2/5.5 G $\left(T_{\mathrm{g}}=85\right)$

PSS1M2/7.0 G $\left(T_{\mathrm{g}}=84\right)$

PSS1M2/8.0 G $\left(T_{\mathrm{g}}=85\right)$

PSS2M $2 / 5.5 \quad \mathrm{G}\left(T_{\mathrm{g}}=85\right)$

PSS2M $2 / 7.0 \quad \mathrm{G}\left(T_{\mathrm{g}}=88\right)$

PSS2M $2 / 10.0 \mathrm{G}\left(T_{\mathrm{g}}=84\right)$

PSC1M2/1.8 GB and K 49.5 $5_{(4.2)}\left(T_{\mathrm{m}}\right) \mathrm{GB}$ and SA $83\left(T_{\mathrm{g}}\right) \mathrm{SA} 157_{(1.7)}\left(T_{\mathrm{c}}\right) \mathrm{I}$

PSC1M2/2.0 GB and K 49.7 $(4.1)\left(T_{m}\right)$ GB and SA $83\left(T_{\mathrm{g}}\right) \mathrm{SA} 157_{(1.5)}\left(T_{\mathrm{c}}\right) \mathrm{I}$

PSC1M2/4.5 GB and K 50.2 $2_{(2.95)}\left(T_{\mathrm{m}}\right) \mathrm{GB}$ and SA $82\left(T_{g}\right) \mathrm{SA} 155_{(0.92)}\left(T_{\mathrm{c}}\right) \mathrm{I}$

PSC1M2/6.0 GB and K 50 $(2.2)\left(T_{m}\right)$ GB and SA $85\left(T_{\mathrm{g}}\right)$ SA $155^{\mathrm{b}}\left(T_{\mathrm{c}}\right) \mathrm{I}$

PSC2M2/5.7 GB and K 50.2 $(3.35)\left(T_{\mathrm{m}}\right) \mathrm{GB}$ and SA $83\left(T_{\mathrm{g}}\right) \mathrm{SA} 155_{(1.27)}\left(T_{\mathrm{c}}\right) \mathrm{I}$

PSC2M2/6.0 GB and K 50.2(3.2) $\left(T_{m}\right)$ GB and SA $83\left(T_{\mathrm{g}}\right) \mathrm{SA} 157_{(0.91)}\left(T_{\mathrm{c}}\right) \mathrm{I}$

PIS1M2/4.5 GB and K $6.9\left(T_{\mathrm{g}}\right) \mathrm{K}$ and SA 52.4 $4_{(2.14)}\left(T_{m}\right) \mathrm{SA} 164_{(0.87)}\left(T_{\mathrm{c}}\right) \mathrm{I}$

PIS2M2/11.0 GB and K $3.2\left(T_{\mathrm{d}}\right) \mathrm{K}$ and SA $56_{(1.85))}\left(T_{m}\right)$ SA $159^{\mathrm{b}}\left(T_{\mathrm{c}}\right) 1$

PIC1M2/4.5 GB and K $6.9\left(T_{\mathrm{g}}\right) \mathrm{K}$ and SA $\left.52.4_{\{2.55)}\left(T_{m}\right) \mathrm{SA} 161_{(0.94}\right)\left(T_{\mathrm{c}}\right) \mathrm{I}$

PIC2M2/8.9 GB and K $1.6\left(T_{\mathrm{g}}\right) \mathrm{K}$ and SA 52.1 $1_{(2.7)}\left(T_{m}\right) \mathrm{SA} 156_{(1.13)}\left(T_{\mathrm{c}}\right) \mathrm{I}$

BAC2M2/3.8 GB and K $13.5\left(T_{\mathrm{g}}\right) \mathrm{K}$ and SA $49.5_{(3.12)}\left(T_{m}\right) \mathrm{J} \mathrm{SA} 157_{(1.44)}\left(T_{\mathrm{c}}\right) \mathrm{I}$

BAC2M2/23 GB and K $34.5\left(T_{\mathrm{g}}\right) \mathrm{K}$ and SA $51_{(1.22)}\left(T_{\mathrm{m}}\right) \mathrm{SA} 151^{\mathrm{b}}\left(T_{\mathrm{c}}\right) \mathrm{I}$

${ }^{a} G$, amorphous glass; $G B$, birefringent glass; $K$, crystallized LC; $S A$, smectic A phase; I, isotropic phase; $T_{g}$, glass transition temperature; $T_{m}$, melting temperature; $T_{c}$, clearing temperature. Transition enthalpy is subscripted in parenthesis ( $\mathrm{kJ} / \mathrm{mol}$ of $L C$ ) to the appropriate transition temperature.

${ }^{b}$ Transition not detected by DSC but observed by POM.

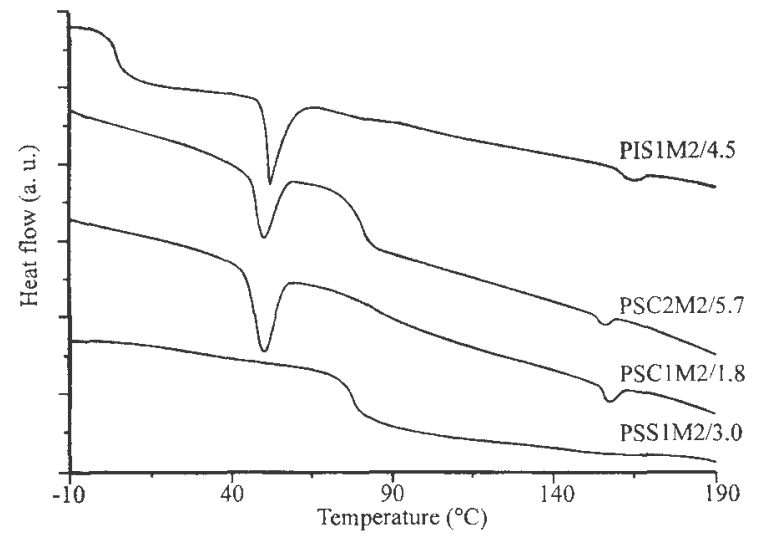

Fig. 1. Typical DSC curves of LCH.

Two different strategies have been considered in order to impart liquid crystallinity to the $\omega$ - and $\alpha, \omega$ sulfonato polystyrenes listed in Table 3 . The first approach consists in adding mesogen molecules in order to fill in the space between two adjacent mesogenic cations strongly associated to the sulfonate anions of the polystyrene chains.

As reported elsewhere, a mesophase is formed when a low molar mass LC is added to the nonmesogenic LC sulfonato (semi)telechelic polystyrenes [27]. When the mesogen excess is high, the smectic mesophase is observed over the same temperature range as the pure mesogen. Thus the mesogen phase separates from the polystyrene matrix as dispersed microdomains. The second strategy relies upon the substitution of a flexible polymer for the polystyrene chains, so that the ionic mesogens become mobile enough for a liquid crystalline order to emerge. Since the mobility of the polymer chains is dramatically increased above the glass transition temperature, a low $T_{g}$ polymer should be advantageously used. The validity of this approach is supported by the PSS1M2/0.7 sample, which 
clearly shows a smectic mesophase in agreement with a very low $T_{g}\left(19^{\circ} \mathrm{C}\right.$ compared to $98-104^{\circ} \mathrm{C}$ for the samples of higher MW, Table 3). An additional evidence may be found in low $\mathrm{T}_{\mathrm{g}} \mathrm{LC} \omega$ - and $\alpha, \omega-$ sulfonato polyisoprenes, whose the liquid crystalline properties are reported in Table 3.

In sharp contrast to the series of LC sulfonato (semi)telechelic polystyrenes, the carboxylato counterparts are mesogenic, all the other conditions being the same. Fig. 2 illustrates the optical texture for some of these samples observed at $120^{\circ} \mathrm{C}$ in a sandwich cell under crossed polarizers. Actually, mesophases were assigned on the basis of the optical textures. For this purpose, each polymer was heated up to approximately $10^{\circ} \mathrm{C}$ above the clearing point and then allowed to cool down slowly through the phase transition. For the low molar mass PSS1M2/1.8, rods are formed at the transition to yield a fan-shaped texture (Fig. 2a) indicative of a layered organization [36,37]. In addition, regions of homeotropic texture are visible, which indicates that the director of the phase is oriented perpendicular to the cell windows. The tendency for ionically functionalized liquid-crystals to align perpendicularly to the glass substrate was previously observed by other research groups $[15,38]$. This homeotropic alignment is disrupted by shearing the sample between the glass plates, and a shimmering aspect is observed. The observed phase was assigned to a smectic A phase, in agreement with the surface alignment of amphiphilic alkylam-monium mesogens, the ionic groups of which acts as a smectogen [15]. The adsorption of ammonium ions on the glass plates triggers the perpendicular alignment of the LC molecules and the formation of a homeotropic structure in which the nitroazobenzene mesogenic groups are able to overlap as the result of the strong mutual interaction of the nitro terminal groups. The homeotropic structure formed in the smectic phase remains unaltered on cooling below $T_{g}$. The oriented domains can accordingly be frozen in the solid state.
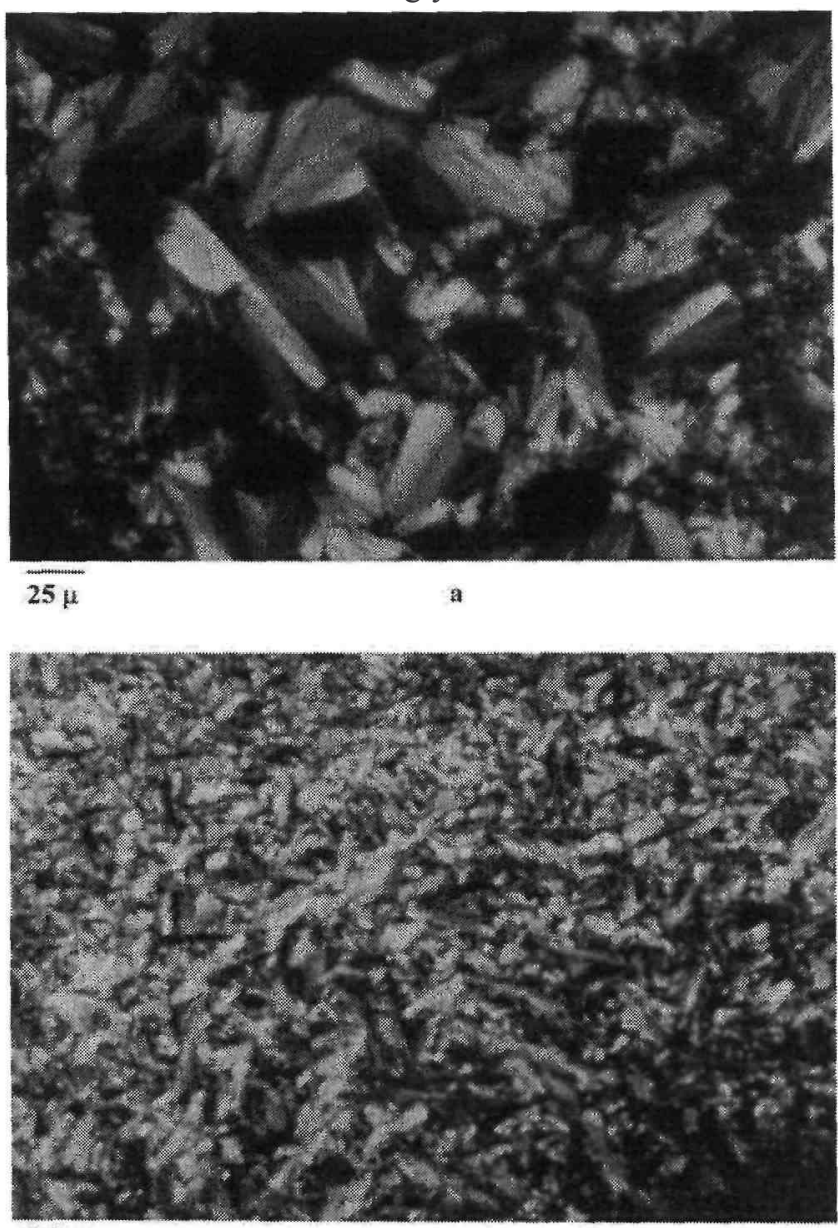

Fig. 2. Optical texture observed for the PSC1M2/1.8 (a), PSC1M2/4.5 (b), PIC1M2/4.5 (c), and $B A C 2 M 2 / 3.8(d)$ samples (crossed polarizers, observation temperature: $100^{\circ} \mathrm{C}$ ). 
This feature is of great interest for many applications such as non-linear optics and data storage [39]. For the higher molecular mass carboxylato LCH (i.e. PSC1M2/4.5, PSC2M2/5.7), the optical texture consists of small domains as shown in Fig. 2b. This texture remains essentially unchanged even after annealing for several hours, which is in favor of a less ordered mesophase. Actually, the mesophase would be the same as for lower mass analog, but perturbed by some defects, in agreement with the low enthalpy of the smectic-isotropic phase transition as observed by DSC (e.g. PSC1M2/1.8 and PSC1M2/4.5; BAC2M2/3.8 and BAC2M2/23.0; Table 3).

The thermal behavior of these samples was studied by DSC and POM. When observed by POM at room temperature, $\mathrm{LC} \omega$ - and $\alpha, \omega$-carboxylato polystyrenes show a birefringent glassy phase (noted as GB in Table 3) that coexists with crystallized LC (K phase in Table 3). A smectic A mesophase is formed at ca. $50^{\circ} \mathrm{C}$, as supported by the observation of the texture characteristic of this type of mesophase (POM) and by a melting endotherm $\left(T_{m}\right.$ in the DSC curve (see PSC1M2/1.8 and PSC2M2/5.7 in Fig. 1).
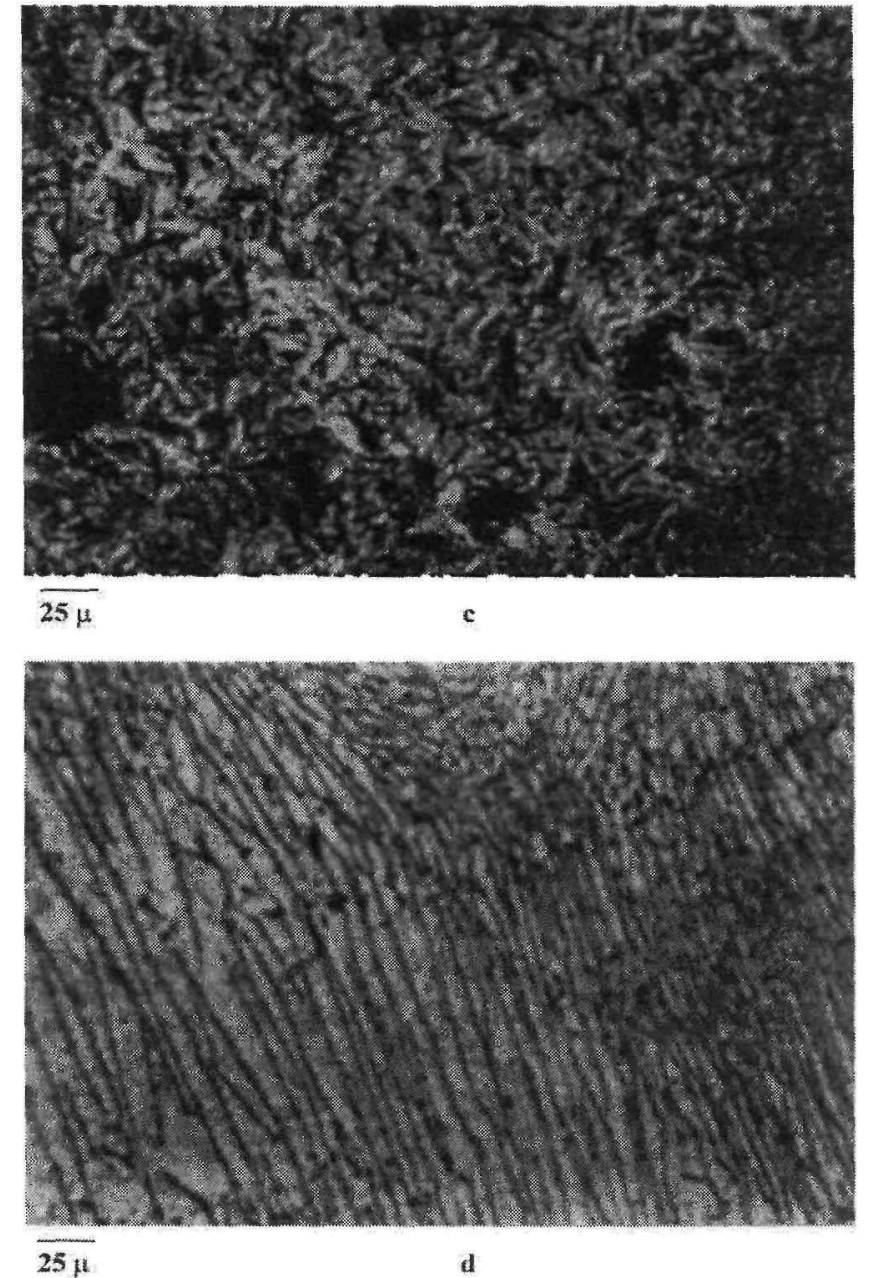

Fig. 2. (continued)

This observation is in complete agreement with Ujiie et al. [15] who studied the liquid crystalline properties of LCPs prepared from poly(sodium styrenesulfonate) and the mesogenic cation M2. However, the careful examination of the POM texture indicates that the SA mesophase still contains some GB phase domains. Therefore, SA and GB phases coexist above the melting temperature of the $\mathrm{K}$ phase, in agreement with a glass transition $\left(\mathrm{T}_{\mathrm{g}}\right.$ ) in the DSC curve (see Table 3 and PSC1M2/1.8 and PSC2M2/ 5.7 in Fig. 1). This complex thermal behavior can only be explained by some residual ionic mesogen M2 in the LC carboxylato (semi)telechelic polystyrenes, as the result of the non-complete ion-exchange between the ammonium halide group of M2 and the alkaline salt end-group of the polymer precursor. The coexistence of free mesogen molecules, free polystyrene chains and mesogen ions associated to the polystyrene chains $(\mathrm{LCH})$ can explain that below the melting temperature of M2 $\left(T_{m}\right), \mathrm{H}$ and $\mathrm{LCH}$ form the GB glassy phase, whereas the residual mesogen forms the crystalline $\mathrm{K}$ 
phase. Above $T_{m}$, the $\mathrm{K}$ phase is melted. The 'free' mesogen molecules are immiscible with the polymer backbone and are expected to migrate in the LC subphase. Above $T_{m}$, a complex material is formed which shows the coexistence of GB and SA phases. This strongly suggests a two-phase material in which the LC molecule are nanophase-separated from the polymer matrix. Indeed, the LC molecules could be arranged in a SA mesophase in the $T_{g}-T_{m}$ temperature range. The polymer backbones being still in the glassy state could impede the formation of well-defined textures. In this respect, the GB phase disappears above $\mathrm{T}_{\mathrm{g}}$ in favor of a well-defined SA mesophase, that persists until the clearing temperature $\left(T_{c}\right)$. Although it may be a problem to detect the smectic-isotropic transition by DSC because of a low enthalpy change, $T_{C}$ is observed by optical microscopy at ca. $155^{\circ} \mathrm{C}$ for all the mesogenic carboxylato LCH. Therefore, the polystyrene molecular weight seems to have no effect on $T_{\mathcal{C}}$, in contrast to the usual increase in the mesomorphic-isotropic transition temperature with molecular weight in conventional LCPs.

At high temperature $\left(>200^{\circ} \mathrm{C}\right)$, thermal degradation occurs, more likely due to the limited thermal stability of the ammonium groups [40].

All the analyzed $\omega$ - and $\alpha, \omega$-carboxylato- and sulfonato-polyisoprene-based LCH are liquid crystalline, as confirmed by DSC and POM. At temperatures below $\mathrm{T}_{\mathrm{g}}$ of the GB phase, this birefringent glassy phase coexists with the $\mathrm{K}$ phase (see Table 3 and PIS1M2/4.5 in Fig. 1). Above $T_{g}$, the GB phase is transformed into a SA mesophase, whereas the $\mathrm{K}$ phase is still observed by POM. An unique SA mesophase is finally formed in the $T_{m}-T_{c}$ temperature range (Table 3 and PIS1M2/4.5 in Fig. 1). Fig. 2c illustrates that quite similar textures are formed by polyisoprene and polystyrene containing $\mathrm{LCH}$. These textures are generally ill-defined and need annealing to be observed.

LC carboxylato-telechelic poly(tert-butylacrylate)s show the same characteristic features as the polyisoprene counterparts (see Table 3). Fig. 2d illustrates the typical texture which is spontaneously formed when LC $\alpha, \omega$-carboxylato poly(tert-butylacrylate)s are annealed at $140^{\circ} \mathrm{C}$ for two hours. A lamellar organization is clearly seen consisting of highly birefringent rows alternating with darker ones. Hyper-structures were previously reported for $\alpha, \omega$-metal carboxylato poly(tert-butylacrylate)s of 21,000 MW and containing $\mathrm{Na}, \mathrm{Mg}, \mathrm{Ca}$ or Ba counterions [41]. An hexagonal supramolecular organization was inferred from a series of X-ray scattering peaks observed at very small angles. By analogy, the typical texture shown by the LC $\alpha, \omega$-carboxylato poly(tert-butylacrylate)s is thought to be related to a specific supramolecular structure.

The liquid crystalline properties of LCH are also controlled by the molecular weight of the polymer backbone. Indeed the LC properties are no longer observed above a molecular weight of ca. 8000 in the case of the LC $\omega$-carboxylato polystyrene, compared to 700 for the LC $\omega$-sulfonato polystyrene. When telechelic polyisoprene (PIS2M2/11.0) and poly(tert-butylacrylate) (BAC2M2/23.0) are used, LC properties persist at higher MW, i.e. 11,000 and 23,000, respectively, although typical textures and endotherms become less detectable. Consistently, the MW of difunctional (telechelic) samples, above which the mesogenic properties disappear, is two times higher compared to the monofunctional (semitelechelic) counterparts. Accordingly, the mesogen can only phase separate above a critical mesogen/ polymer weight ratio, that however changes with the polymer. It is not surprising that this critical weight ratio is higher in the case of a covalently bonded LCP compared to the model ionic counterparts [28], as result of more severe constraints for the mesogen. Therefore the ionic bonding of the mesogen, particularly at the chain extremities, is an efficient way to decrease the mesogen content required for inducing the mesogen phase separation.

Finally, Table 3 shows that $T_{m}\left(49.5-56^{\circ} \mathrm{C}\right)$ and $T_{c}\left(151-164^{\circ} \mathrm{C}\right)$ are basically independent of the polymer backbone, the ionic end-groups (carboxylate or sulfonate) and the polymer MW. So, the mesophase stability is essentially dictated by the LC, although the stability range is less extended compared to the pure LC.

It is now clear that the liquid crystalline properties of LCH are controlled by the nature of both the ionic end-group(s) and the polymer, and by the length of the polymer chains.

\section{Small-angle X-ray scattering and small-angle neutron scattering studies of LCH}

Nanophase-separation of the polymer segments and the mesogenic molecules was reported in Section 3 , and nanoscale-organization, such as smectic A mesophase, as well. Therefore, formation of supramolecular structures cannot be precluded, which could be detected by SAXS and SANS, provided that strong enough contrast exists between the constitutive nanophases.

At this point of the discussion, it must be recalled that each constitutive component of LCH has a characteristic supramolecular organization. The morphology of the H precursors, that was extensively 
studied by SAXS [42-44], is dominated by the microphase separation of the ionic end-groups from the polymer matrix $[45,46]$. The ionic microdomains, known as multiplets, are small $(6-9 \mathrm{~A})$ and the average interdistance is dictated by the polymer MW, in agreement with the position of a main diffraction peak (designated as the ionic peak), which results from interparticle interferences [46]. Moreover, a small-angle intensity upturn is systematically observed, whose the origin was attributed to large scale heterogeneities throughout the material. Finally, a long-range organization was reported for $\alpha, \omega$-carboxylato poly(tert-butylacrylate)s in a possible relation to the large cross-section of the polymeric backbone compared to the ion pair [41].

The ionic mesogen M2 forms a smectic mesophase, which was characterized by Ujiie et al. [15]. In a smectic A mesophase, the LC molecules are stacked into layers, with no particular order between molecules within the layer. In SAXS or SANS experiment, the interlayer distance can be calculated form a Bragg diffraction peak arising from interlayer reflection. An interlayer distance of $3.48 \mathrm{~nm}$ was calculated by Ujiie et al. from wide-angle X-ray diffraction measurements on M2 [15].

Fig. 3 shows the SANS curves of the mesogen M2, the H precursor PSS1 Li/0.7 and the LCH PSS1M2/ 0.7. Diffraction peaks are observed for all the samples which indicate that the neutronic contrast is large enough, although no deuteration was carried out. In the SANS profile of the PSS1Li/0.7, a diffraction peak, which was attributed to the ionic peak, is observed at $3.3 \mathrm{~nm}$. The strong intensity upturn at small scattering vectors is also observed in this sample. The SANS curve for M2 is characterized by a diffraction peak at $3.4 \mathrm{~nm}$, which was attributed to the smectic layer peak in complete agreement with the results reported by Ujiie et al. [15]. In LCH, a mesogenic counterion is substituted for the metal one. In contrast to metal counterions, mesogenic ones are able to organize themselves with formation of mesophases. Furthermore, since the mesogenic ions tend to pack into layers, the size and shape of the multiplets is expected to be strongly modified. This aspect will be discussed in Section 6, in which a detailed study of the multiplets organization will be discussed. The SANS profile for the PSS1M2/0.7 LCH is shown in Fig. 3. Substitution of a mesogenic counterion for the metal one is responsible for a deep modification in the supramolecular organization of the original halato(semi)-telechelic polymer. Two peaks are observed, which correspond to the ionic peak at $6.8 \mathrm{~nm}$ and to the layer spacing peak at $3.4 \mathrm{~nm}$, respectively. The characteristic distance associated to the ionic peak is much larger in the LCH $(6.8 \mathrm{~nm})$ compared to the H precursor $(3.3 \mathrm{~nm})$. This modification is thought to result from the bulkiness of the mesogenic ions and from the different structure of the multiplets formed by mesogenic ion pairs. 
Published in: Progress in polymer science (2001), vol. 26, iss. 7, pp. 1061-1099 Status: Postprint (Author's version)

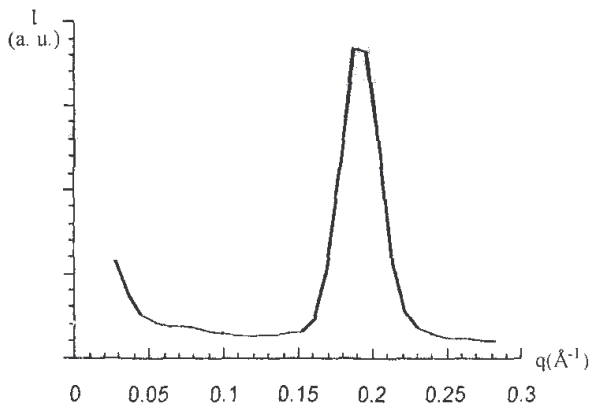

$\mathbf{a}$

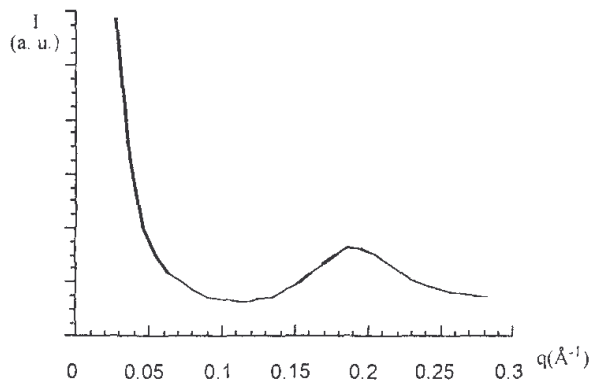

b

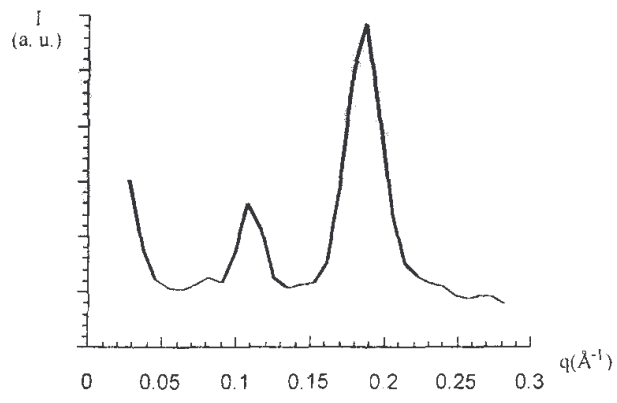

c

Fig. 3. SANS profiles for the M2 (a), PSS1Li/0.7 (b), and PSS1M2/0.7 (c) samples.

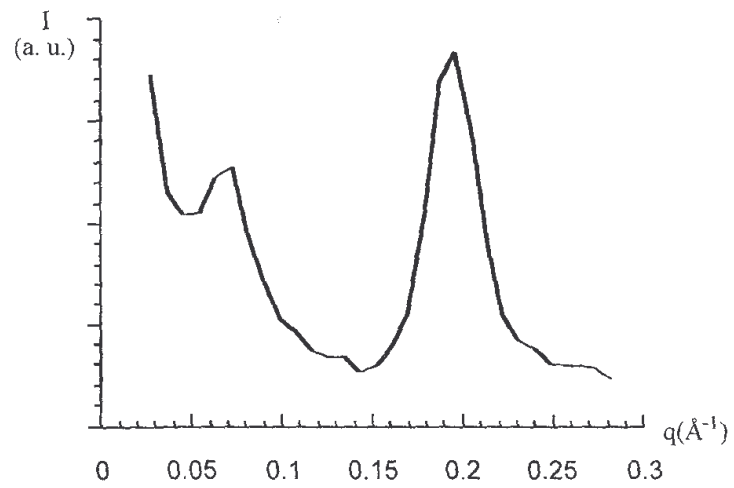

Fig. 4. SANS profile for the PSC1M2/2.0 sample. 
The layer spacing peak, which will be now referred to as the 'mesogenic peak', is observed at the same position as the layer spacing peak of M2, in agreement with the existence of smectic A mesophases of the same structure in M2 and PSS1M2/0.7. The observation of both the ionic peak and the mesogenic peak in the PSS1M2/0.7 sample will be discussed in Section 5.

The SANS curve of the PSC1M2/2.0 sample is shown in Fig. 4. The ionic peak and the mesogenic peak are once again simultaneously observed in this sample but the intensity of the ionic peak is much lower in this sample than in the PSS1M2/0.7 sample. The mesogenic peak is located at the same position as before $(3.4 \mathrm{~nm})$ while the distance associated to the ionic peak is larger $(8.3 \mathrm{~nm})$ in the PSC1M2/2.0 sample than in the PSS1M2/0.7 (6.8 $\mathrm{nm})$.

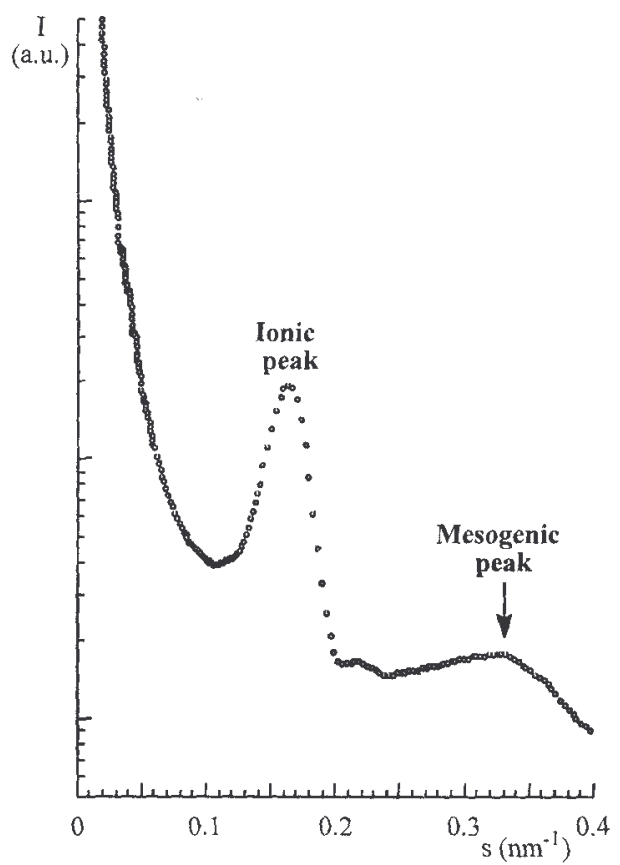

Fig. 5. SAXS profile for the PSS1M2/0.7 sample.

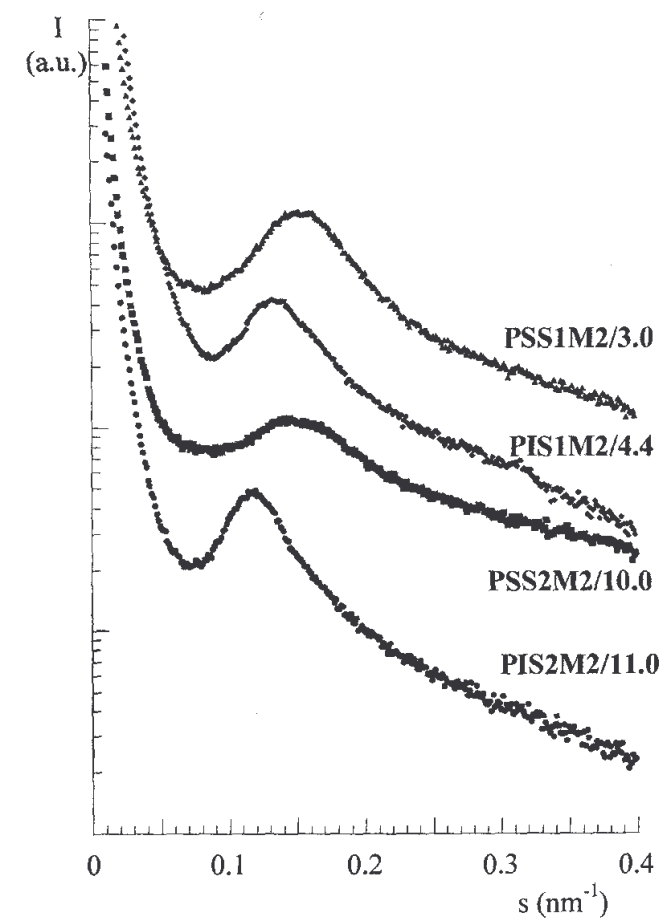

Fig. 6. Effect of the polymer backbone on the SAXS profiles. SAXS intensity has been multiplied by an arbitrary factor, such that the curves do not overlap each other. 
This effect is thought to essentially result from an increased molecular weight in the PSCIM2/2.0 sample, which tends to push the mesogenic multiplets away one from each other.

SAXS experiments were extensively conducted on LCH and compared with the SANS data for the same samples. In this respect, the SAXS curve for the PSS1M2/0.7 sample is shown in Fig. 5 and shows distinct peaks at ca. 6.3 and $3.2 \mathrm{~nm}$, respectively. These two peaks were assigned to the ionic peak and the mesogenic peak, respectively. In the SAXS curve, the relative intensity of the ionic peak is much larger than for the mesogenic peak, the reverse being observed for the SANS curve. Moreover, the position of the ionic and the mesogenic peaks is slightly different in the SAXS and the SANS curves because of the different origin of the contrast in SAXS and SANS experiments. Fluctuations in electronic density are indeed at the origin of the contrast in SAXS experiments. The sulfur atoms of sulfonato LCH are thus thought to play a key role in the SAXS contrast, which will be accordingly weak in carboxylato LCH. In SANS experiments, the contrast is due to fluctuations in the neutronic cross-sections, thus a characteristic feature of the nuclei and no longer of the electrons of the constitutive atoms [47]. In this respect, the nitrogen atoms of the mesogenic ions should be mainly responsible for the SANS contrast.

SAXS curves of LCH were compared in order to probe the dependence of the characteristic features of the SAXS profiles on various molecular parameters, such as chemical nature of the polymer backbone and the end-group(s), and mono- or difunctionality of the chains.

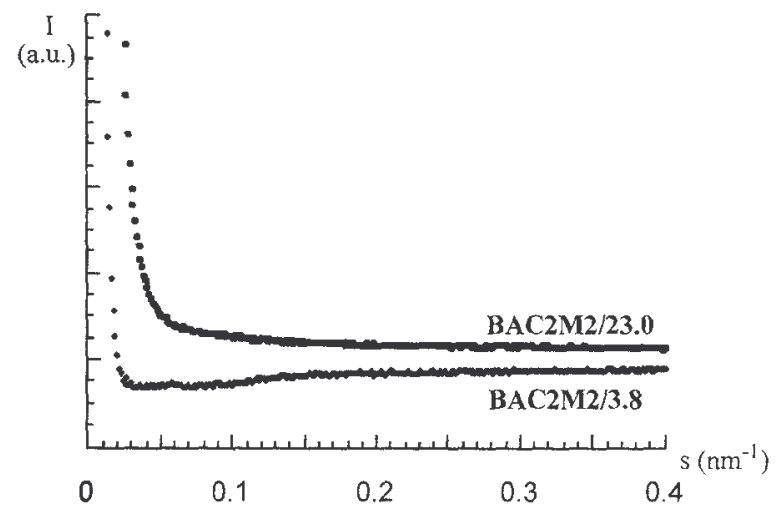

Fig. 7. SAXS profiles for two poly(tert-butylacrylate)-based LCH.

Fig. 6 compares the SAXS profiles and the ionic peak position, $s$ IP, for LC $\omega$ - and $\alpha, \omega$-sulfonato polystyrenes and LC $\omega$ - and $\alpha, \omega$-sulfonato polyisoprenes. For the samples shown in Fig. 6 , the small difference in $M_{n}$ of polystyrene and polyisoprene chains cannot explain that the characteristic distance $\mathrm{d}_{\mathrm{IP}}(=1 / \mathrm{sIP})$ is systematically larger for the polyisoprene-based samples ( 7.57 and $8.48 \mathrm{~nm}$ for the PIS1M2/4.4 and PIS2M2/11.0 samples, respectively) compared to the polystyrene ones (6.44 and 6.83 $\mathrm{nm}$ for the PSS1M2/3.0 and PSS2M2/10.0 samples, respectively). The reason for this difference has rather to be found in the actual degree of polymerization which is higher for polyisoprene at a constant MW and in the 1,4-units of the polyisoprene chains.

The SAXS curves of poly(tert-butylacrylate) containing samples are shown in Fig. 7. Surprisingly enough, the ionic peak and the mesogenic peak are no longer observed, which is tentatively explained by a too weak electronic contrast.

Fig. 8 interestingly compares $\omega$ - and $\alpha, \omega$-LC sulfonate polystyrenes. At the same MW, dIP is $15-20 \%$ smaller for the telechelic polystyrene compared to the semi telechelic analog. For instance, dIP is 7.36 and $7.76 \mathrm{~nm}$ for the PSS1M2 samples of $\bar{M}_{n}=5500$ and 7000, respectively, compared to 6.09 and $6.75 \mathrm{~nm}$ for the PSS2M2 counterparts. This observation is in complete agreement with data previously reported for $\mathrm{H}$ polystyrenes [48].

Fig. 9 is a comparison of LC $\omega$-carboxylato- and sulfonate polystyrene and polyisoprene. As a rule, the ionic peak intensity is significantly smaller for the carboxylate containing samples than for the sulfonate counterparts, to the point where the ionic peak can be unobserved in the case of short PS chains end-capped by a carboxylate group (PSC1M2/1.8). Although a decrease in the electronic contrast when carboxylate moieties are substituted for sulfonate one may be part of the explanation, it is surprising that no ionic peak is observed for a short PS chain $\left(\bar{M}_{n}=1800\right)$ end-capped by one mesogenic carboxylate group, because this peak is unambiguously detected in the case of a 
polyisoprene chain of a twofold MW $\left(\bar{M}_{n}=4500\right)$ end-capped by the same group (PIC1M2/4.5). Furthermore, a mesogenic peak is observed for these two samples (PSC1M2/1.8 and PIC1M2/4.5). These observations will be discussed in Section 5 .

The effect of temperature on the SAXS intensity was also examined. At $45^{\circ} \mathrm{C}$, the PSS1M2/0.7 sample shows a dramatic decrease in the ionic peak intensity compared to $30^{\circ} \mathrm{C}$ (Fig. 10). This intensity goes on decreasing at higher temperatures, and the ionic peak is no longer observed at $100^{\circ} \mathrm{C}$.

In contrast, the intensity of the broad mesogenic peak slightly increases with temperature. These observations are in agreement with the macroscopic liquid crystalline character of the PSS1M2/0.7 sample (Table 3), which is confirmed now by the persistency of the mesogenic peak. The vanishing intensity of the ionic peak upon increasing temperature could be due to the increased mobility of the mesogenic ions with temperature. As a result, the position of the ionic peak should become less defined and the intensity lower.

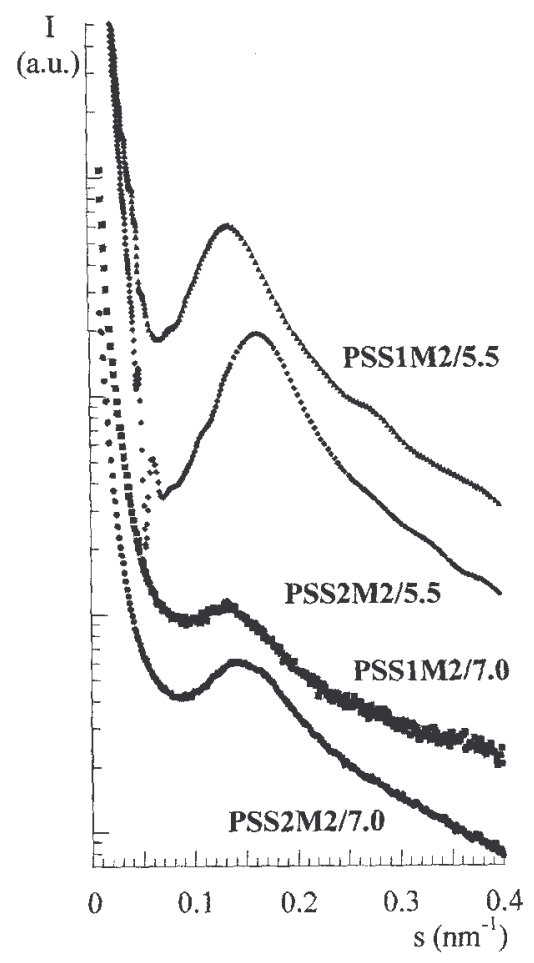

Fig. 8. Comparison of the SAXS profiles for LC $\omega$ - and $\alpha, \omega$-sulfonato polystyrene of the same $M W$. SAXS intensity has been multiplied by an arbitrary factor, such that the curves do not overlap each other.

\section{Model for the supramolecular organization of LCH}

The experimental observations reported in the previous sections can be explained on the basis of a morphological model for the $\mathrm{LCH}$, which relies on two driving forces: the dipolar association of the ion pairs in multiplets, as it occurs in ionomers, and the supramolecular organization of the mesogens in smectic mesophases. The signatures of these supramolecular structures are the ionic peak for the multiplet organization and the mesogenic peak for the smectic organization, respectively, as schematized in Fig. 11. The multiplet structure is shown in Fig. 11a, the ionic cores containing now bulky mesogenic ions. A detailed analysis of the multiplet organization will be given in Section 6. The smectic organization is essentially the same as the layered structure of the smectic A mesophase found in M2. The mesogenic ions form layers in which the individual molecules are interdigitated [15]. The polymer chains are not miscible and microphase separate from the mesogenic ions, that leads to a sandwich structure with the polymer chains located in the interlayer spacing (Fig. $1 \mathrm{lb}$ ). 


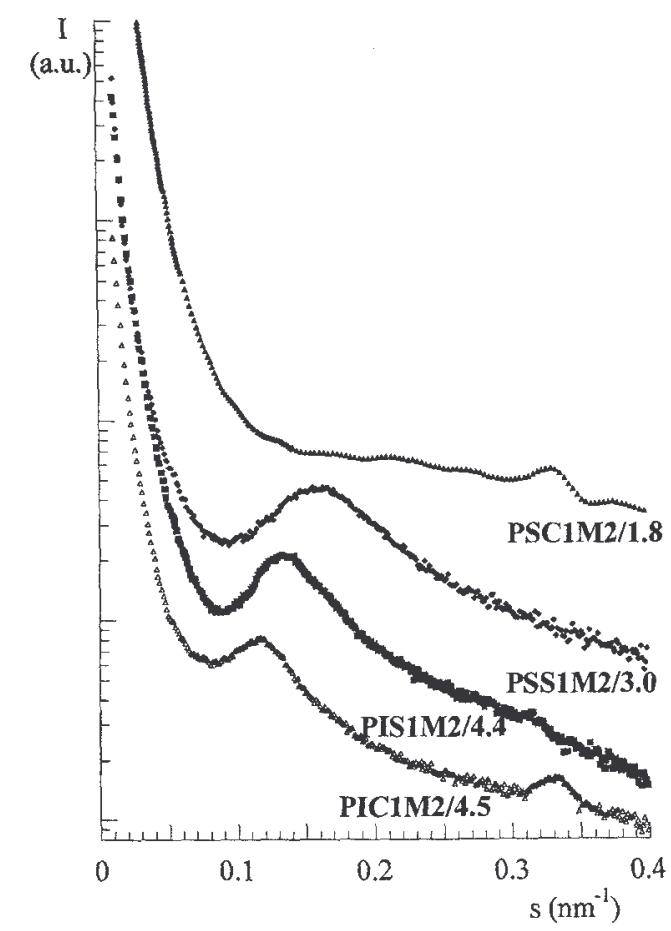

Fig. 9. Effect of the anionic end-group on the SAXS profiles. SAXS intensity has been multiplied by an arbitrary factor, such that the curves do not overlap each other.

Depending on the nature and MW of the polymer backbone, the nature of the ion pairs and temperature, one type of organization will dominate or both of them will coexist. Each of these extreme situations is illustrated in Fig. 9: only the mesogenic peak is observed in PSC1M2/1.8 (high $\mathrm{T}_{\mathrm{g}}$ polystyrene and weak dipolar interactions), only the ionic peak is observed in PSS1M2/3.0 (high -g polystyrene and strong dipolar interactions), whereas the two peaks coexist in PIC1M2/4.5 (low $T_{g}$ polyisoprene and weak dipolar interactions).

The strength of the mutual electrostatic interactions of the ion pairs (i.e. dipoles in a apolar environment) is the driving force for the multiplet formation, in a closer relation to the size of the constitutive ions and the ionicity of the bond between them. If these electrostatic interactions are too weak to overcome the elastic forces of the chains to which they are attached, no multiplet is formed. In this respect, strong electrostatic interactions are operative in ammonium sulfonate ion pairs compared to carboxylate ones. It is well known from the scientific literature on ionomers that the mutual dipolar interactions of metal sulfonates largely exceed the interactions of metal carboxylates, all the other conditions being the same. For instance, Lundberg and Makowski [49] and Rigdahl and Eisenberg [50] concluded from rheological studies that the effect on physical properties is several orders of magnitude larger for sulfonate ion pairs than for carboxylate ones. Indeed, the softening behavior of the metal sulfonate ionomer suggests that strong ionic association persists at temperatures higher by $50-100^{\circ} \mathrm{C}$ compared to the carboxylate ionomer. 


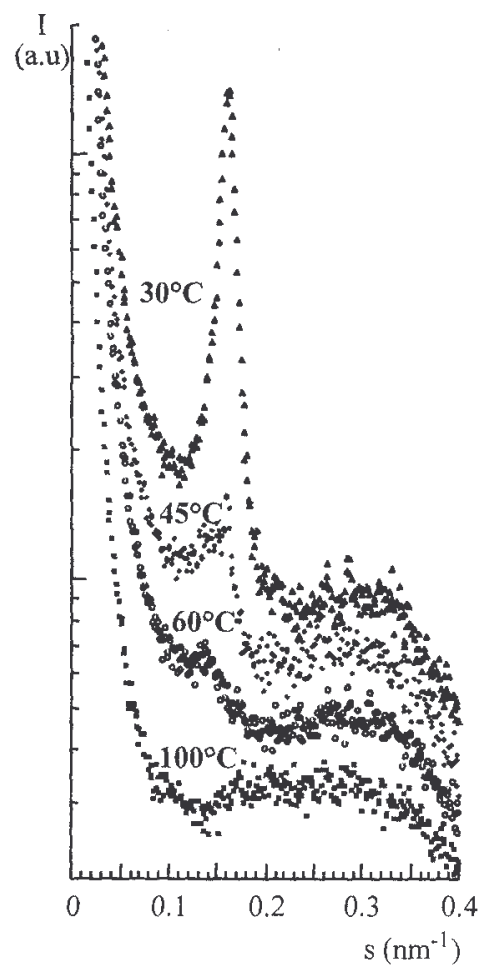

Fig. 10. Effect of temperature on the SAXS profile for the PSS1M2/0.7 sample. SAXS intensity has been multiplied by an arbitrary factor, so that the curves do not overlap each other.

Similarly, the melt viscosity of sulfonate ionomers is two or three orders of magnitude larger than the carboxylate analogs (same ion pair content).

From the molecular point of view, Lefelar and Weiss [51] proposed models for the structure of metal sulfonate and metal carboxylate ion pairs on the basis of a crystal close packing approach. These models show that the anion packing of carboxylates depends on the counterion, in sharp contrast to the packing of the sulfonate anions which is completely independent of the cation.

Fig. 12 is a comparison of the ionic close packing of the sulfonate and the carboxylate anions associated with a monovalent cation, in this case a mesogenic quaternary ammonium cation. Fig. 12a shows how the oxygen atoms of the carboxylate anions are organized in two parallel hexagonally packed layers, with one layer slightly displaced with respect to the other one. In Fig. 12a, the mesogenic cations fit into the six-coordinated trigonal-bypyramidal hole between the anion layers, as it occurs in $\mathrm{NaH}\left(\mathrm{CH}_{3} \mathrm{CO}_{2}\right)$ [52].

This packing scheme is consistent with the structure of a smectic mesophase. Ions can be further added possibly in any direction of this planar array, so as to form long-range ordered layers of densely packed mesogen molecules. In this situation, the distance between two near neighbor mesogen molecules is small enough for the liquid crystalline order to be stabilized by intermolecular interactions. Fig. 12b shows the antiparallel overlapping of layers of hexagonally packed sulfonate anions with the mesogenic cations fitting the octahedral holes limited by the anion edges which correlates with the published structure for $\mathrm{Na}_{2}\left(\mathrm{CH}_{2}\left(\mathrm{SO}_{3}\right)_{2}\right)$ [52]. 

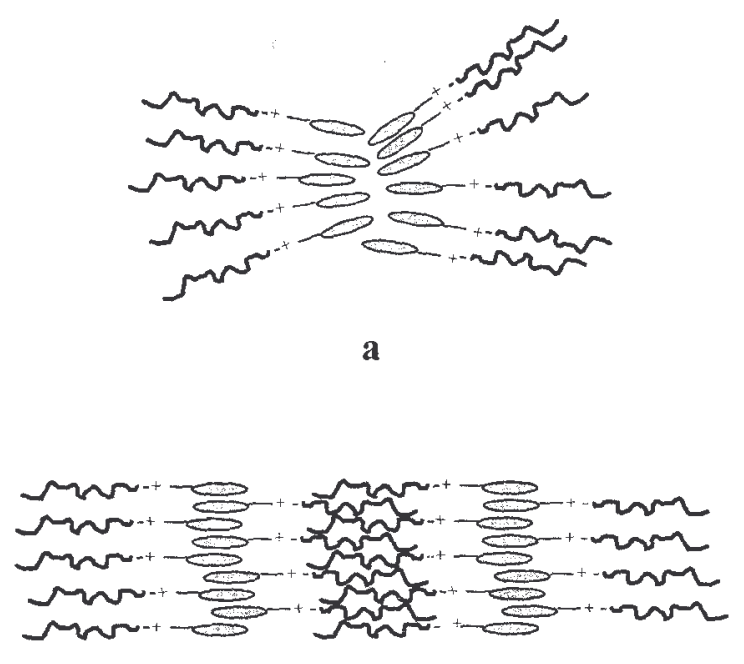

b

+ : M2 molecule

Fig. 11. Sketches of the multiplets (a), and smectic (b) supramolecular organizations.
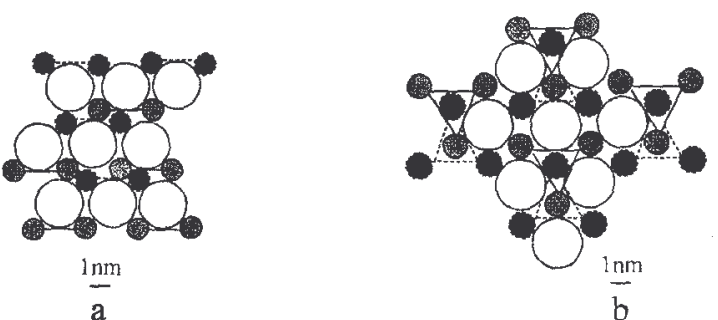

Fig. 12. Close packing in carboxylate (a), and sulfonate LCH (b). Large open circles are ammonium cations; and small filled circles are oxygen atoms below the cation plane. Small shaded circles are oxygen atoms above the cation plane. Lines connect oxygen atoms from the same carboxylate or sulfonate anion.

As for the carboxylate analogs, additional ion pairs can approach in any direction. The distance between two adjacent mesogens in however larger compared to the carboxylate anion array. Furthermore, the mesogenic cations are more strongly associated with the sulfonate $\mathrm{H}$, which restricts their mobility and accordingly their ability to form a mesophase. The combination of these two effects can prevent the sulfonato LCH from being organized in a smectic mesophase.

So, it is not surprising that sulfonate polystyrene based LCH are not mesogenic and tend to be organized into multiplets, as confirmed by the observation of only one ionic peak in these systems (Figs. 5 and 8). Liquid crystallinity is however observed when a flexible polymer is substituted for the polystyrene chains.

In this line, the low $\mathrm{T}_{\mathrm{g}}$ PSS1M2/0.7 and sulfonato(semi)telechelic polyisoprenes form mesophases as shown by DSC and POM and by a smectic layer peak in the SAXS profile (Fig. 9). However, an ionic peak persists in the SAXS profile so that combination of strong enough dipolar interactions and polymer of low $T_{g}$ allows for smectic domains and ionic multiplets to coexist.

An additional valuable information has to be found in the temperature dependence of the SAXS curves. When the temperature is increased, the intensity of the ionic peak decreases in contrast to the intensity of the mesogenic peak which increases (Fig. 10). This observation agrees with a gradual transition from multiplets to smectic mesophases.

The formation of mesophases is usually observed in the carboxylato LCH considered in this study, as illustrated by the PSC1M2/1.8 sample (Fig. 9), which only shows the mesogenic peak. Ionic multiplets and smectic domains can also coexist at room temperature in carboxylato LCH (PIC1M2/4.5 in Fig. 9). Expectedly, the mesogen/polymer weight ratio has a decisive effect on the final morphology, the 
mesophase formation being as favorable as this ratio is high. This observation can be rationalized on the basis of the model of Fig. 11. Indeed, the polymer chains are assumed to fill the interlayer spacing of the smectic mesophase. Since this smectic organization is characterized by one layer spacing of ca. $3.4 \mathrm{~nm}$, the maximum space available for the polymer chains is $3.4 \mathrm{~nm}$. The chain conformation must accommodate this critical value for the smectic organization to emerge. Above a critical molecular weight, which depends on the chemical nature of the polymer, this requirement is no longer fulfilled and the smectic organization is lost.

\section{Multiplet organization and restriction of mobility in LCH}

A general model for the phase morphology of ionomers was proposed by Eisenberg, Hird and Moore (EHM model) [46], according to which the multiplets restrict the mobility of the chain segments directly attached to them. When the concentration of the ion pairs is high enough, these regions of restricted mobility come into contact and coalesce to form clusters. As a result, an additional and higher glass transition temperature is observed. Experimental evidence for the restricted mobility of chain segments adjacent to the ionic multiplets was found by NMR analysis of partially deuterated telechelic polystyrenes [53,54], by measurement of the dielectric relaxation of telechelic polyisoprenes [55] and by EPR analysis of random ionomers [56]. However, little attention has been paid so far to the thickness of the region of restricted mobility. According to the EHM model, this restriction mobility would extend over ca. the persistence length of the polymer, thus over a few angstroms.

The purpose of this section is to show that SAXS is a valuable technique to measure the thickness of the region of restricted mobility in sulfonato-semitelechelic polystyrene containing a mesogenic counterion, thus for these $\mathrm{LCH}$ whose the supramolecular organization has been found to be dominated by the multiplet organization.

The SAXS patterns of the LC sulfonato-semi telechelic polystyrenes are shown in Fig. 13. The ionic peak is systematically observed, which is the signature of the dipolar association and phase separation of the ion pairs, which contain in this case a mesogenic counterion. The average distance between the ionic microdomains (dIP) can be extracted from the position of the ionic peak. Consistently, dIP increases with the polystyrene molecular weight whatever the counterion, mesogenic in the LCH series and metallic ( $\mathrm{Li}$ ) in the $\mathrm{H}$ series (Table 4). Moreover, dIP is larger for the LCH samples than for the $\mathrm{H}$ precursors. The origin of a larger average distance between the ionic domains in LCH has something to do with the substitution of the mesogenic cation for the metallic one in $\mathrm{H}$, as already noted in Section 4. The mesogenic peak is only observed in the PSS1M2/0.7 sample, as previously discussed.

The precise geometry of the multiplets formed by the mesogenic ion pairs is a pending question. If the size of the mesogenic multiplets is assumed to be of the order of magnitude of the smectic layer spacing, the distance between two near neighbor microdomains cannot be smaller than ca. $3.5 \mathrm{~nm}$. In other words, dIP should be the result of two contributions: the length of the polymer backbone and the size of the mesogenic ion. 


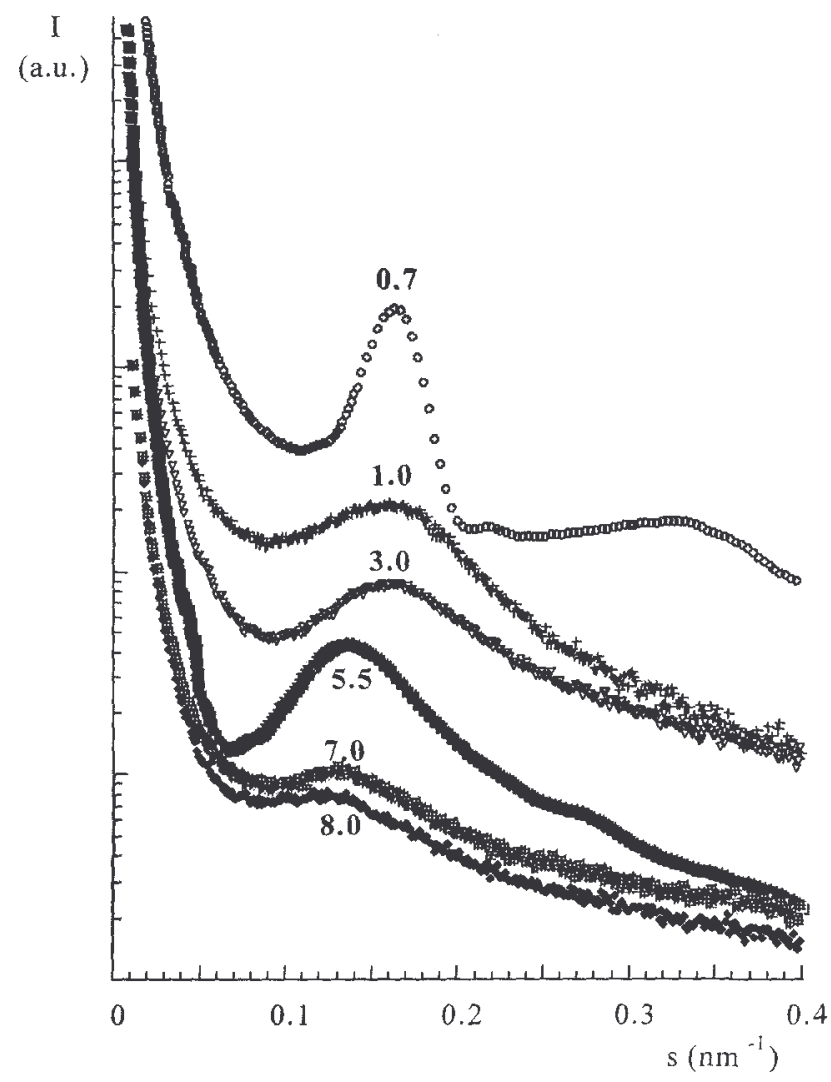

Fig. 13. SAXS curves of sulfonato polystyrene LCH in bulk (each curve has been indexed by the $\bar{M}_{n}$ of the corresponding $\mathrm{LCH}$ ).

Table 4 Distance associated to the ionic peak of LCH and the parent precursors

\begin{tabular}{|c|c|c|c|c|c|}
\hline Sample & $\begin{array}{l}\mathrm{d}_{\text {IP }} \mathrm{LCH} \\
(\mathrm{nm})\end{array}$ & $\begin{array}{l}\mathrm{d}_{\mathrm{IP}} \\
\mathrm{LCH} /<\mathbf{r}_{0}{ }^{2}>^{1 / 2 \mathrm{a}}\end{array}$ & $\begin{array}{l}\mathrm{d}_{\text {IP }} \mathrm{H} \\
(\mathrm{nm})\end{array}$ & $\begin{array}{l}\mathrm{d}_{I \mathrm{P}}> \\
\mathrm{H} /<\mathrm{r}_{0}{ }^{2}>^{1 / 2 \mathrm{a}}\end{array}$ & $\begin{array}{l}\mathrm{d}_{\text {IP }} \mathrm{H} \\
(\mathrm{nm})^{\mathrm{b}}\end{array}$ \\
\hline PSS1M2/0.7 & 6.06 & 3.36 & 3.3 & 1.82 & 2.99 \\
\hline PSS1M2/1.0 & 6.32 & 2.92 & 3.44 & 1.57 & 3.33 \\
\hline PSS1M2/3.0 & 6.25 & 1.68 & 4.63 & 1.24 & 4.67 \\
\hline PSS1M2/5.5 & 7.36 & 1.46 & 5.82 & 1.11 & 5.63 \\
\hline $\mathrm{PSS} 1 \mathrm{M} 2 / 7.0$ & 7.76 & 1.31 & 6.22 & 1.06 & 6.06 \\
\hline $\mathrm{PSS} 1 \mathrm{M} 2 / 8.0$ & 7.6 & 1.24 & 6.27 & 1.03 & 6.31 \\
\hline
\end{tabular}

${ }^{a}$ The bulkiness of the multiplet is not taken into account. ${ }^{b}$ Calculated from Eq. (1).

However, the very simple relationship that emerges ( $\mathrm{d}_{\mathrm{IP}} \mathrm{LCH}=\mathrm{d}_{\mathrm{IP}} \mathrm{H}_{\text {precursor }}+$ thickness of the smectic layer spacing) is not valid to the samples under consideration in this paper. In order to clear up this observation, the experimental data have been compared to previous SAXS data reported for $\alpha, \omega-$ sulfonato polystyrenes containing a metal cation [48]. Important information on the structure is provided by the dependence of the peak position, $\mathrm{d}_{\mathrm{IP}}$, on the polymer molecular weight. Indeed, a three-dimensional isotropic packing of spherical multiplets should give distances changing with the cubic root of the polymer molecular weight. For a two-dimensional packing of rod-like multiplets, the dependence should be the square root of molecular weight and, for a one-dimensional stacking of lamellae, it should be linear with molecular weight.

As far as bulk metal sulfonato-semitelechelic polystyrenes are concerned, the following power law is found for dIP [48]:

$$
d_{\mathrm{IP}}=0.4\left(\bar{M}_{n}\right)^{\alpha}, \quad \alpha=0.307( \pm 0.02)
$$

The experimental value of $\alpha$ (close to $1 / 3$ ) is thus in favor of the isotropic organization of the ionic multiplets, which strongly suggests that an increase in molecular weight pushes the multiplets away from each other while keeping unchanged the structure and space distribution. Substitution of a 
mesogenic cation for the metal one has a deep effect, since the experimental data in Table 4 no longer fit Eq. (1), indicating that the spatial organization of the multiplets is also deeply modified. Therefore, the experimental results (Table 4) have been fitted to the two-dimensional rod-like model. As shown in Fig. $14, d_{\mathrm{IP}} / \bar{M}_{n}^{1 / 2}$ does not depend linearly on $\bar{M}_{n}^{1 / 2}$. The one-dimensional stacking of lamellae is neither suited to describe the phase morphology of LCH, the dependence of $d_{\mathrm{IP}} / \bar{M}_{n}$ on $\bar{M}_{n}$ being not linear.

The discrepancy between the experimental data and the three models could originate from the bulkiness of the mesogenic core, whose the thickness should be subtracted from $d_{l P}$ before testing the validity of the possible power laws. On the assumption that the thickness of the mesogenic core is the same for all the samples under consideration, i.e. $3.5 \mathrm{~nm}$, the constancy of $d_{I P} /\left(\bar{M}_{n}\right)^{\bar{\beta}}(\beta=1 / 3,1 / 2$ or 1$)$ is not restored in LCH when $\left(\mathrm{d}_{\mathrm{IP}}-3.5 \mathrm{~nm}\right.$ ) is used rather than $\mathrm{d}_{\mathrm{IP}}$. (see for example $\mathrm{d}_{\mathrm{IP}}$ $3.5 / \bar{M}_{n}^{1 / 2}$ versus

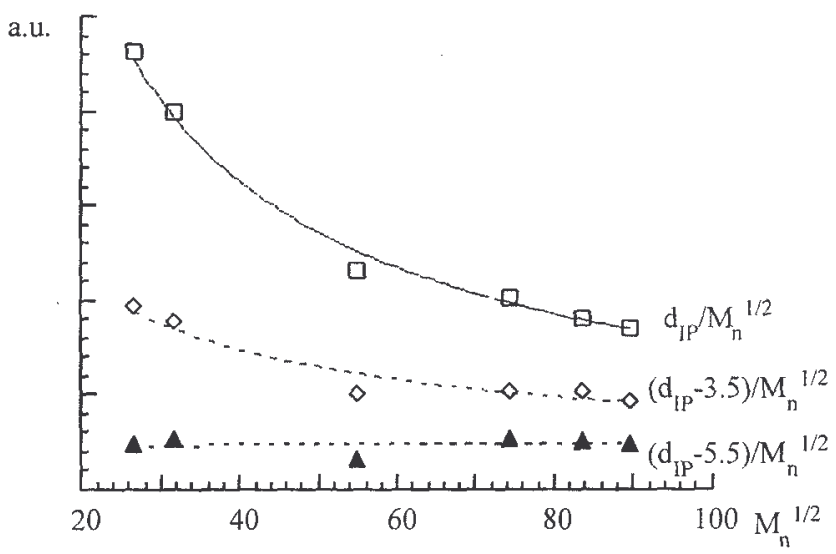

Fig. 14. Dependence of $d_{\mathrm{IP}} / \bar{M}_{n}^{1 / 2}$ on $\vec{M}_{n}^{1 / 2}$ in sulfonate polystyrene $L C H$.

$$
\begin{aligned}
& \bar{M}_{n}^{1 / 2} \text { in Fig. 14) } \\
& \left(\bar{M}_{n}\right)^{1 / 2}=14.6\left[\left(r_{0}\right)^{2}\right]^{1 / 2}
\end{aligned}
$$

As an additional piece of information, the calculation of the unperturbed end-to-end distance of polystyrene from Eq. (2) [57] shows that the polystyrene chains are in a conformation as more extended as the molecular weight is small (Table 4). This observation suggests that the multiplets formed by the mesogenic sulfonates are responsible for a restriction of mobility and thus for the local extension of the directly connected polymer segments. This effect is consistent with the strong mutual electrostatic interactions of the sulfonate ion pairs $[49,50]$ and could explain that no liquid crystalline properties are usually observed in LC $\omega$-sulfonato polystyrene samples (Section 2). According to the EHM model, the thickness of the region of restricted mobility is of the order of the persistence length of the polymer chain which is in the range of $1 \mathrm{~nm}$ for polystyrene chains [46]. In this respect, $\mathrm{d}_{\mathrm{IP}}$ in LC $\omega$-sulfonato polystyrenes should rather result from three contributions: the length of the polystyrene chain, the thickness of the mesogenic multiplets and the length of the stretched polystyrene segments attached to multiplets (Fig. 15). Therefore, the constancy of $d_{\mathrm{IP}} /\left(\bar{M}_{n}\right)^{\beta}(\beta=1 / 3,1 / 2$ or 1$)$ should be restored in $\mathrm{LCH}$ if $\left(\mathrm{d}_{\mathrm{IP}}-5.5 \mathrm{~nm}\right)$ is considered rather than $\mathrm{d}_{\mathrm{IP}}$ or $\left(\mathrm{d}_{\mathrm{IP}}-3.5 \mathrm{~nm}\right)$. 


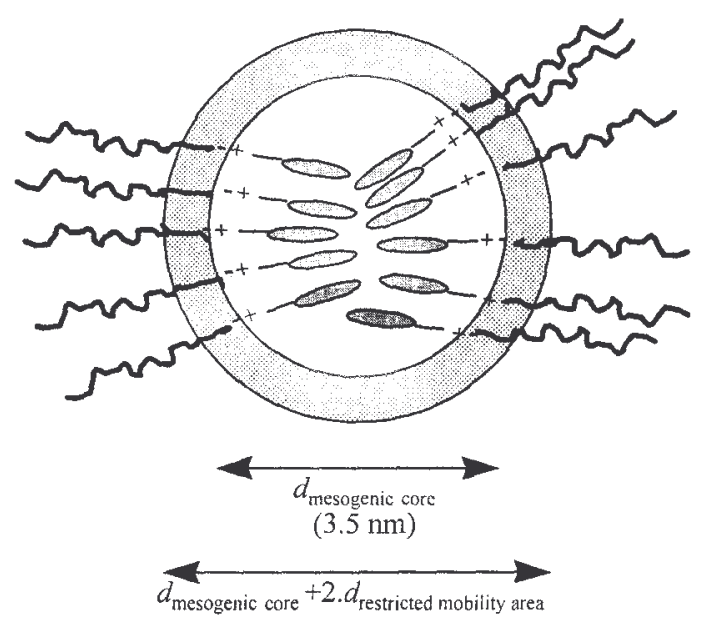

$(3.5+2 \times 1=5.5 \mathrm{~nm})$

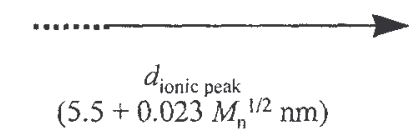

+ : Mesogenic ion

n . : Sulfonato polystyrene

$\sqrt{6}:$ : Restricted mobility area

Fig. 15. Restricted mobility area in sulfonato polysytrene LCH.

In this respect it appears that $(\mathrm{d} I \mathrm{P}-5.5 \mathrm{~nm}) / \bar{M}_{n}^{\beta}$ is independent of $\bar{M}_{n}^{\beta}$ when $\beta=1 / 2$ (see Fig. 14):

$$
d_{\mathrm{IP}}-5.5=0.023\left(\bar{M}_{n}\right)^{\beta}, \quad \beta=0.5( \pm 0.02)
$$

Therefore, the experimental data are in agreement with the theoretical model proposed by Eisenberg et al. together with a rod like structure for the spatial organization of the mesogenic ion pairs.

Solutions of LC $\omega$-sulfonato polystyrenes in toluene were analyzed by SAXS in a large concentration (C) range from 10 to $60 \mathrm{wt} \%$. Beyond $60 \mathrm{wt} \%$, the polymer is no more soluble in toluene. The experimental data are summarized in Table 5. The scattering maximum is continuously shifted towards lower s values as the dilution is increased.

If the phase morphology is assumed to remain unchanged upon dilution by an apolar solvent, Eq. (5) should be valid to solutions of sulfonate $\mathrm{LCH}$ polystyrenes:

$\mathrm{d}$ IP $-5.5=K C^{-1 / 2}$

However, from the data calculated in Table 5, the actual dependence of $d_{w}$ versus $C$ is rather:

$d_{l P}-5.5=K^{\prime} C^{\delta}, \quad \delta=-1 / 3( \pm 0.03), \quad K^{\prime}=8.82 \mathrm{~nm}(\mathrm{wt} \%)^{1 / 3}$

So, when LCH are dissolved in toluene, the supramolecular organization of the multiplets is deeply modified and agrees with a transition from a rod-like structure in bulk to an isotropic distribution of the multiplets in concentrated toluene solution. The organization of the LC $\omega$-sulfonato polystyrenes in toluene is quite similar to the $\mathrm{H}$ precursors. In this respect, solutions of sulfonato $\mathrm{H}$ were previously analyzed by Vanhoorne et al. [48], who found that dIP depends on the polymer concentration according to a power law in agreement with an isotropic distribution of the multiplets:

$\mathrm{d}_{\mathrm{IP}}=K^{\prime \prime} C^{\varepsilon}, \quad \varepsilon=-0.33( \pm 0.02) \quad$ (6)

In sharp contrast to the $\mathrm{H}$ parent compounds, the presence of a region of restricted mobility in solution of LCH must also be taken into account since $\left(\mathrm{d}_{\mathrm{IP}}-5.5 \mathrm{~nm}\right)$ is the actual length directly dependent on $\tilde{M}_{n}$ and not dIP nor (dIP - $3.5 \mathrm{~nm}$ ). So, the region of restricted mobility in LCH is not affected by the solvent which indicates that the polymer chain segments in the vicinity of the multiplets are not solvated in LCH.

This could be related to the observed poor solubility of $\mathrm{LCH}$ in toluene compared to the parent $\mathrm{H}$. Once 
again the substitution of a mesogenic ion for a metallic one deeply affects the physico-chemical properties of the final material both in bulk and in solution.

Table 5 Distance associated to the ionic peak of PSS1M2/5.5 in toluene solution

$\begin{array}{lllll}\begin{array}{l}\text { Concentra } \\ \text { tion }(\mathrm{C},\end{array} & \begin{array}{l}\mathrm{d} \text { IP }-5.5 \\ \mathrm{nt} \%)\end{array} & \begin{array}{l}K^{\prime} C^{-1 / 3}(\mathrm{~nm}) ; K^{\prime}=8.82 \\ \mathrm{~nm}(\mathrm{wt} \%)^{1 / 3}\end{array} & \begin{array}{l}L C^{-1 / 2}(\mathrm{~nm}), \\ \mathrm{L}=19 \mathrm{~nm}(\mathrm{wt} \%)^{1 / 2}\end{array} & \begin{array}{l}M C^{-1}(\mathrm{~nm}), M=190 \mathrm{~nm} \\ (\mathrm{wt} \%)\end{array} \\ 100 & 1.9 & 1.9 & 1.9 & 1.9 \\ 54.9 & 2.1 & 2.32 & 2.56 & 3.46 \\ 51.5 & 2.2 & 2.37 & 2.65 & 3.69 \\ 36.5 & 2.5 & 2.66 & 3.14 & 5.2 \\ 25.3 & 3 & 3 & 3.77 & 7.51 \\ 21.6 & 3.1 & 3.17 & 4.08 & 8.8 \\ 14.5 & 3.6 & 3.62 & 4.99 & 13.1 \\ 12.5 & 3.7 & 3.8 & 5.37 & 15.2\end{array}$

\section{Comparison of LCH prepared by either ion-exchange or proton-transfer reaction}

The LCH considered in the previous sections were prepared by ion-exchange of the quaternary ammonium group attached to the M2 mesogen for the metal counterion of $\omega$ - $($ or $\alpha, \omega-)$ metal carboxylate or metal sulfonate polymers.

A second pathway to ionic LCPs is the proton transfer from an acid (on the polymer) to a base (on the mesogen). The completeness of the proton transfer depends however on the relative pica's of the acid and the base involved. The aim of this section is to compare the properties of some LCH prepared by ion-exchange and proton transfer from acid- $\mathrm{H}$ to aminated mesogen. M2 and M4 were used for the synthesis of LCH by ion-exchange, and M1 and M3 were engaged in proton transfer reaction for the synthesis of LCH. The liquid crystalline properties of the final polymers were compared on the basis of DSC analysis, POM and SAXS.

\subsection{Differential scanning calorimetry and polarized optical microscopy analysis}

The thermal properties of some LCH containing M1 and M2, analyzed by DSC and POM, are listed in Table 6. These samples generally show a complex thermal behavior as previously discussed in Section 3.

There is a sharp difference between the first and second scan for the samples prepared by acid-base reaction, in contrast to the DSC curves for the samples prepared by ion-exchange that remain essentially unchanged even when the scanning is repeated several times.

Furthermore, except for the second scan of the PSS1M1/5.5 and PIS2M1/11.0 samples, the transition temperatures for the samples prepared by acid-base reaction are significally different compared to the same LCH samples prepared by ion-exchange reaction. This discrepancy is thought to reflect an incomplete proton-transfer from the acid group of the polymer to the amine of the mesogen. According to the DSC measurements, the proton-transfer is only complete when sulfonic acid derivatives are used, which is line with a much lower $p K a$ than the carboxylic acids. These observations are also in complete agreement with he data recently published by Bazuin and Tork, who studied the liquid crystalline properties of LCPs formed by an aminated non-mesogenic molecule complexed by polyacrylic acid (proton-transfer) compared to with assemblies formed by the ionic non-mesogenic parent molecule with sodium poly(vinylsulfonate) (ion-exchange) [19]. This work proved that liquid crystalline order could be generated from an amorphous polymer and a non-mesogenic molecule. The extent of this ordering was considerably more important for systems containing sulfonates rather than carboxylates moieties. In the present work, the miscibility enhancement observed for the sulfonate-based systems can be so high that the mesogenic properties disappear (e.g. PSS1M1/5.5 and PSS1M2/5.5 samples, Table 6). This effect was discussed in Section 4 and attributed to the huge restriction of mobility experienced by the mesogen molecules when sulfonate ion pairs are concerned [30].

The reaction products of molecules M3 and M4 with halato(semi)telechelic polymers are not mesogenic, whatever the nature of the polymer and the preparation method, although Bazuin and coworkers have reported the possibility of forming LCPs from non-mesogenic components [19]. The thermal properties of the M3 and M4 containing samples are listed in Table 7. Two general situations are observed by DSC and POM. In the first one, a glass transition is systematically observed by DSC for all the samples containing M4 and also for the M3 containing samples but only for the second run 
(Table 7). POM observations indicate that all these samples form an optically clear glass at temperatures below $T_{g}$.

Table 6 Thermal properties of the M1 and M2 containing $L C H$

Acronym

temperatures ${ }^{\mathrm{a}}\left({ }^{\circ} \mathrm{C}\right)$
First scan

$\operatorname{PSS} 1 \mathrm{M} 1 / 5.5$

PIS2M1/11.0

PIC1M1/4.5

BAC2M1/3.8

PSS1M2/5.5

PSC1M2/4.5

PIS2M2/11.0

$\mathrm{PIC} 1 \mathrm{M} 2 / 4.5$

BAC2M2/3.8

$\left(T_{\mathrm{c}}\right) \mathrm{I}$

$\left(T_{\mathrm{c}}\right) \mathrm{I}$

I

$\left(T_{\mathrm{c}}\right) \mathrm{I}$

$\left(T_{\mathrm{c}}\right) \mathrm{I}$

$152(0.87)\left(T_{\mathrm{c}}\right) \mathrm{I}$

I

$\left(T_{\mathrm{c}}\right) \mathrm{I}$

$$
\begin{aligned}
& \mathrm{GB} \text { and } \mathrm{K} 41_{(0.65)}\left(T_{m}\right) \mathrm{GB} \mathrm{G}\left(T_{\mathrm{g}}=85\right) \\
& \text { and SA } 85\left(T_{\mathrm{g}}\right) \text { SA } 125^{\mathrm{b}}
\end{aligned}
$$

GB and K 62 ${ }_{(1.12)}\left(T_{m}\right)$ GB GB and K 60 $(0.81)\left(T_{m}\right)$ GB and SA $83\left(T_{\mathrm{g}}\right) \mathrm{SA}$ and SA $86\left(T_{\mathrm{g}}\right)$ SA $119^{\mathrm{b}} \quad 122^{\mathrm{b}}\left(T_{\mathrm{c}}\right) \mathrm{I}$

GB and K $4\left(T_{\mathrm{g}}\right) \mathrm{K}$ and SA $\quad \mathrm{GB}$ and $\mathrm{K} 13.5\left(T_{\mathrm{g}}\right) \mathrm{K}$ and SA $55_{(1.74)}\left(T_{m}\right) \mathrm{SA}$ $57_{(1.85)}\left(T_{\mathrm{m}}\right) \mathrm{SA} 141^{\mathrm{b}}\left(T_{\mathrm{c}}\right) \quad 157^{\circ}\left(T_{\mathrm{c}}\right) \mathrm{I}$

GB and $\mathrm{K} 0\left(T_{\mathrm{g}}\right) \mathrm{K}$ and $\mathrm{SA}$

${ }^{64}(2.1)\left(\mathrm{T}_{\mathrm{m}}\right) \mathrm{SA} 138_{(0.45)}$

GB and K $4.5\left(T_{\mathrm{g}}\right) \mathrm{K}$ and SA $61_{(2.4)}\left(T_{m}\right) \mathrm{SA}$

$142_{(0.56)}\left(T_{\mathrm{c}}\right) \mathrm{I}$

GB and K $16\left(T_{\mathrm{g}}\right) \mathrm{K}$ and SA GB and K $14.8\left(T_{\mathrm{g}}\right) \mathrm{K}$ and SA $57_{(2.14)}\left(T_{m}\right) \mathrm{SA}$

${ }^{59}(2.32)\left(T_{m}\right) \mathrm{SA} 141_{(0.65)}{ }^{144_{(0.74)}\left(T_{\mathrm{c}}\right) \mathrm{I}}$

$$
\mathrm{G}\left(T_{\mathrm{g}}=85\right) \quad \mathrm{G}\left(T_{\mathrm{g}}=85\right)
$$

GB and $\mathrm{K} 50.8_{(3.65)}\left(T_{\mathrm{m}}\right) \quad \mathrm{GB}$ and $\mathrm{K} 50.2_{(2.95)}\left(T_{m}\right)$ GB and SA $82\left(T_{\mathrm{g}}\right)$

$\mathrm{GB}$ and SA $82\left(T_{\mathrm{g}}\right) \mathrm{SA} \quad \mathrm{SA} 155_{(0.92)}\left(T_{\mathrm{c}}\right) \mathrm{I}$

GB and K $2.8\left(T_{\mathrm{g}}\right) \mathrm{K}$ and SA GB and K $3.2\left(T_{\mathrm{g}}\right) \mathrm{K}$ and SA $56_{(1.85)}\left(T_{m}\right) \mathrm{SA}$

$$
57_{(1.75)}\left(T_{m}\right) \mathrm{SA} 161^{\mathrm{b}}\left(T_{\mathrm{c}}\right) \quad 159^{\mathrm{b}}\left(T_{\mathrm{c}}\right) \mathrm{I}
$$

GB and K $6.7\left(T_{\mathrm{g}}\right) \mathrm{K}$ and SA GB and $\mathrm{K} 6.9\left(T_{\mathrm{g}}\right) \mathrm{K}$ and SA 52.4 $(2.55)\left(T_{m}\right) \mathrm{SA}$ $53_{(2.55)}\left(T_{m}\right) \mathrm{SA} 161_{(1.1)} \quad 1_{(0.94)}\left(T_{\mathrm{c}}\right) \mathrm{I}$

GB and $\mathrm{K} 12.2\left(T_{\mathrm{g}}\right) \mathrm{K}$ and $\quad \mathrm{GB}$ and $\mathrm{K} 13.5\left(T_{\mathrm{g}}\right) \mathrm{K}$ and SA $49.5(3.12)\left(T_{m}\right)$ $\mathrm{SA} 49.5_{(3.25)}\left(T_{m}\right) \mathrm{SA} \quad \mathrm{SA} 157_{(1.44)}\left(T_{\mathrm{c}}\right) \mathrm{I}$ $158_{(1.24)}\left(T_{\mathrm{c}}\right) \mathrm{I}$

${ }^{a} G$, amorphous glass; $G B$, birefringent glass; $K$, crystallized LC; $S A$, smectic A phase; I, isotropic phase; $T_{g}$, glass transition temperature; $T_{m}$, melting temperature; $T_{c}$, clearing temperature. The assignment of the $G, K, G B, S A$ and I phases is based on POM observations. Transition enthalpy is subscripted in parenthesis ( $\mathrm{kJ} / \mathrm{mol}$ of $L C$ ) to the appropriate transition temperature.

${ }^{b}$ Transition not detected by DSC but observed by POM.

In reference to the previous discussion on M1 and M2 containing samples, this behavior should result from strong electrostatic interactions between the M3 and M4 molecules and the carboxylato and sulfonate end-groups, and thus from complete miscibility of the system. In sharp contrast to the M1 and M2 carboxylato end-groups, strong interactions are operative in the M3 and M4 carboxylato containing samples. Therefore, a small change in the structure of the counter-anion can deeply change the strength of the interactions.

The second situation is characteristic of the M3 containing samples before annealing, a birefrin-gent glass and a crystalline phase coexisting at low temperature (PSC1M3/4.5, Table 7). This complex phase is comparable to the coexistence of the GB and K phases in the M1 and M2 containing samples. 
Above the glass transition temperature, a birefringent phase containing some crystalline domains is observed (B and $\mathrm{K}$ phases). An isotropic liquid-like phase is finally formed at temperatures higher than $T_{c}$. When the samples are cooled down, they form an isotropic glassy phase. This thermal behavior is in agreement with a partial proton-transfer form the acid to the amine which tends to completion upon annealing.

Table 7 Thermal properties of the M3 and M4 containing $\mathrm{LCH}$

Acronym Phase transition temperatures ${ }^{\mathrm{a}}\left({ }^{\circ} \mathrm{C}\right)$

First scan Second scan

PSS1M3/5.5 GB and K $97.9\left(\mathrm{~T}_{\mathrm{g}}\right) \mathrm{K}$ and B 154.7 $(3.18)\left(\mathrm{T}_{\mathrm{c}}\right) \mathrm{I} \quad \mathrm{G}\left(T_{\mathrm{g}}=-97.4\right)$

PSC1M3/4.5 GB and K $94.3\left(T_{\mathrm{g}}\right) \mathrm{K}$ and B $165_{(2.67)}(T.) \mathrm{I} \quad \mathrm{G}\left(T_{\mathrm{g}}=86.7\right)$

PIS2M3/11.0 GB and K $21.8\left(T_{\mathrm{g}}\right) \mathrm{K}$ and B 159.4(2.99) $\left(\mathrm{T}_{\mathrm{c}}\right) \mathrm{I} \mathrm{G}\left(T_{\mathrm{g}}=18\right)$

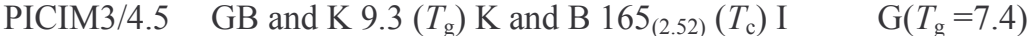

BAC2M3/3.8 GB and K $20.7\left(T_{\mathrm{g}}\right) \mathrm{K}$ and B 163.2(3.44) $\left(T_{\mathrm{c}}\right) \mathrm{I} \quad \mathrm{G}\left(T_{\mathrm{g}}=26.7\right)$

PSS1M4/5.5 G $\left(T_{\mathrm{g}}=100.2\right) \quad \mathrm{G}\left(T_{\mathrm{g}}=100.5\right)$

PSC1M4/4.5 G $\left(T_{\mathrm{g}}=81.9\right) \quad \mathrm{G}\left(T_{\mathrm{g}}=91.8\right)$

PIS2M4/11.0 G $\left(T_{\mathrm{g}}=16.5\right) \quad \mathrm{G}\left(T_{\mathrm{g}}=19.6\right)$

PIC1M4/4.5 G $\left(T_{\mathrm{g}}=9.4\right) \quad \mathrm{G}\left(T_{\mathrm{g}}=9.5\right)$

BAC2M4/3.8 G $\left(T_{\mathrm{g}}=36.7\right) \quad \mathrm{G}\left(T_{\mathrm{g}}=35.4\right)$

${ }^{a} G$, amorphous glass; $G B$, birefringent glass; $K$, crystal phase; $B$ unidentified birefringent phase; $I$, isotropic phase; $T_{g}$, glass transition temperature; $T_{c}$, clearing temperature. The assignment of the $G, K$, $B, G B$ and 1 phases is based on POM observations. Transition enthalpy is subscripted in parenthesis ( $\mathrm{kj} / \mathrm{mol}$ of $L C$ ) to the appropriate transition temperature.

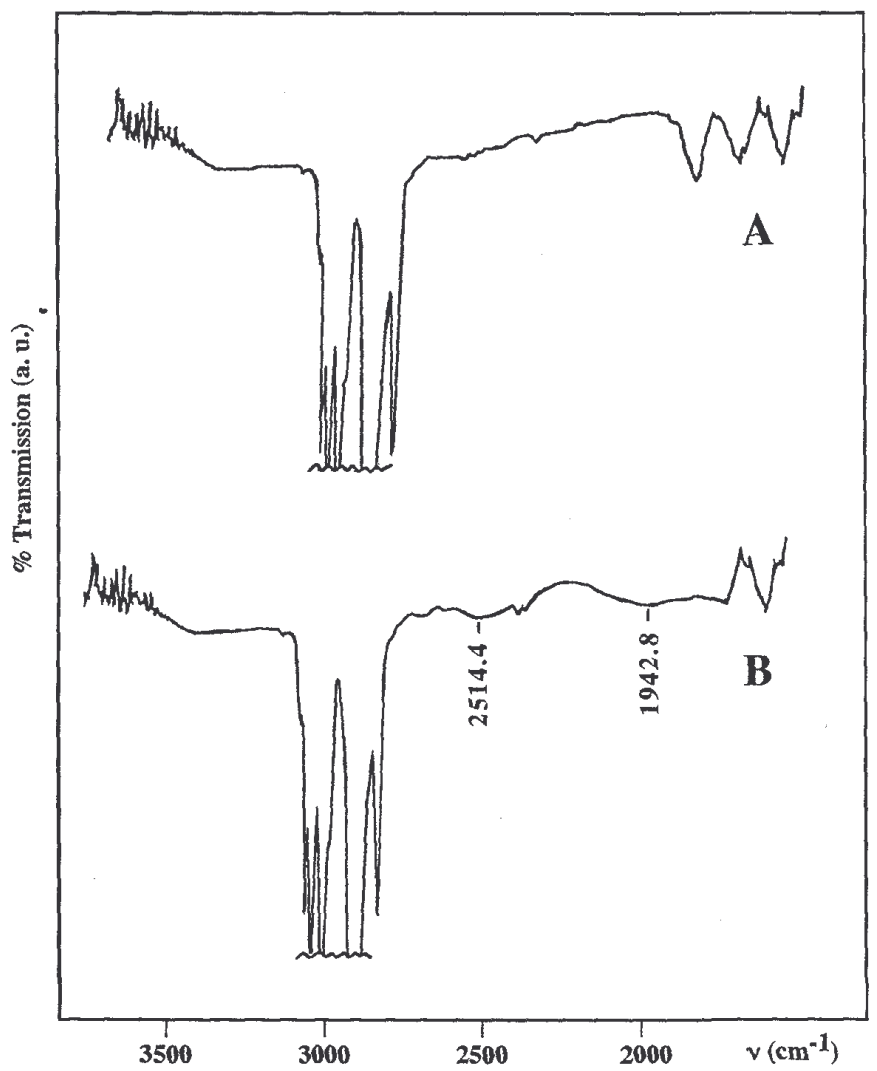

Fig. 16. FTIR spectra for PSC1H/4.5 (A) and PSC1M1/4.5 (B). 


\subsection{Fourier-transform infrared spectroscopy analysis}

The interactions of the polymer end-group(s) with the M1 and M3 molecules was analyzed by FTIR. Fig. 16 compares the FTIR spectra for the carboxylic acid precursor (e.g. PSC1H/4.5) and mixture of it with the non-ionic mesogen M1 (PSC1M1/4.5). Hydrogen bond formation is supported by two new broad absorptions at 2500 and $1950 \mathrm{~cm}^{-1}$ for the mesogen/polymer mixture (PSC1M1/4.5) in agreement with previously reported data $[58,59]$ in the case of strong hydrogen-bonding between carboxylic acid and amine groups. Furthermore, the acid carbonyl absorption at ca. $1700 \mathrm{~cm}^{-1}$ is observed to slightly decrease in favor of the absorption of the carboxylate anion at ca. $1550 \mathrm{~cm}^{-1}$ when the sample is annealed, in agreement with the partial proton-transfer from the carboxylic acid group to the amine. Therefore, the phase transitions for the annealed (second scan) LC carboxylato (semi)telechelic polymers are observed at temperatures intermediate between the values observed for the non-annealed sample and for the parent sample prepared by ion exchange (Table 6). These results are also in qualitative agreement with data reported by Tal'roze et al. who concluded to the formation of hydrogen bonds with strong proton polarization in mixtures of polycarboxylic acid and aliphatic tertiary amines $[60,61]$.

A nearly complete proton-transfer from polyacid to aliphatic tertiary amine was however noted by Bazuin et al. [19]. The reaction progress was followed by disappearance of the acid carbonyl absorption in favor of the carboxylate one. Fig. 17 confirms a dramatic decrease in the carboxylic acid absorption in favor of the carboxylate one upon annealing of the PSC1M3/4.5 sample. Moreover, no hydrogen-bonding is clearly detected in the spectrum.

This difference can be related to the pKa's of diethylamine and diethanolethylamine, respectively. Diethylamine is a good model for M3, and diethanolamine for M1. p-Ka values (11.09 for diethylamine and 8.96 for diethanolamine, at $\left.25^{\circ} \mathrm{C}\right)$, clearly indicate that diethylamine is a stronger base than dietha-nolethylamine.

Therefore, proton-transfer is expected to be much more effective when M3 is used rather than M1. Finally, no hydrogen-bonding between the hydroxyl moieties of the M1 molecule and the acid group of the polymer can be suspected by FTIR.

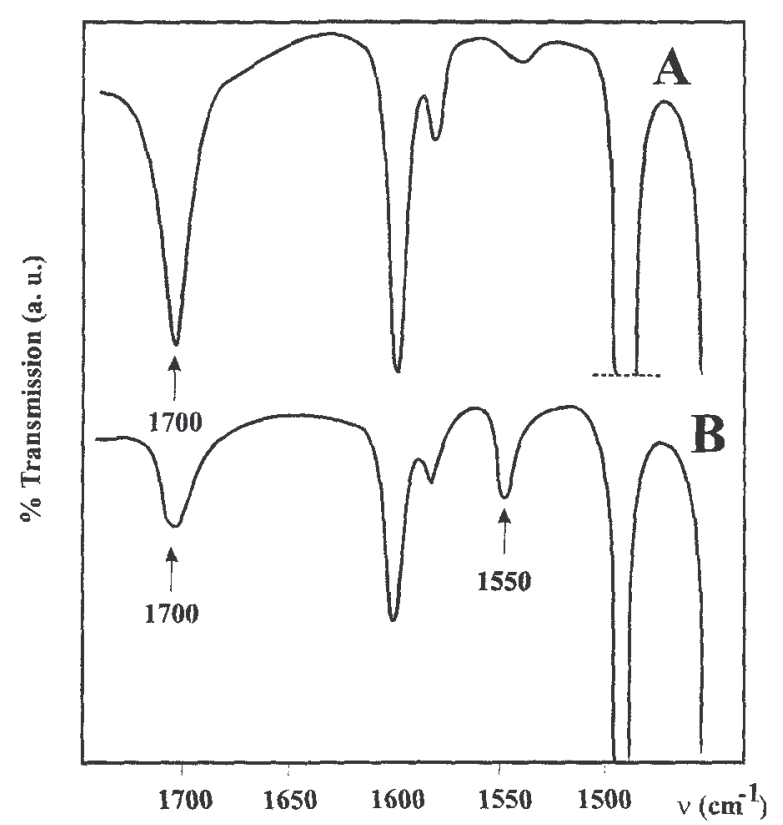

Fig. 17. FTIR spectra for PSC1M3/4.5 before (A) and after annealing (B). 


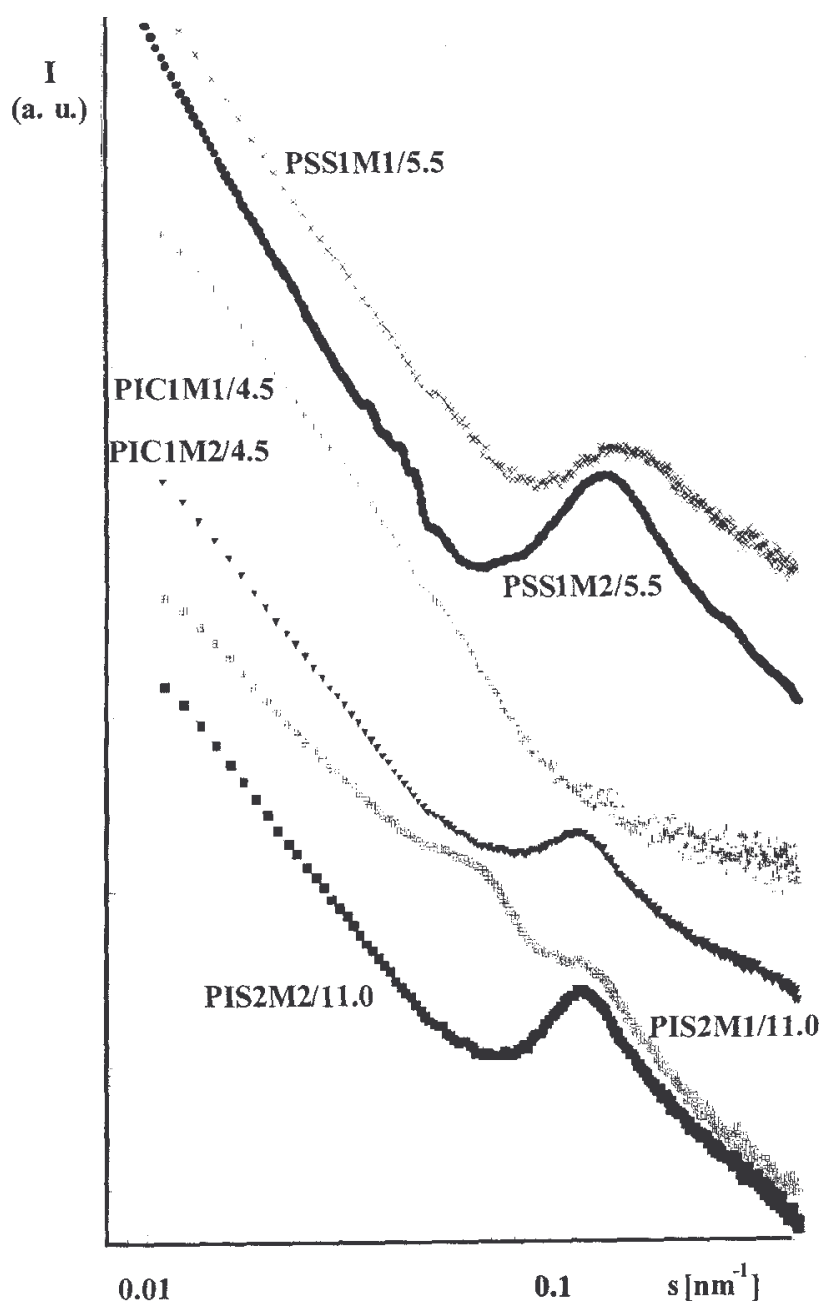

Fig. 18. SAXS curves for annealed M1 and M2 containing LCH.

\subsection{Small-angle X-ray scattering analysis}

The SAXS curves for LCH containing the M1 and M2 LC and prepared by either ion-exchange or acidbase reaction are shown in Fig. 18. Most of the SAXS curves show an ionic peak, in agreement with the dominant multiplet organization in these materials. The proton-transfer is at least part from the sulfonic acid to the amine group since an ionic peak is observed for the PSS1M1/5.5 and PIS2M1/11.0 samples prepared by the so-called acid-base method. Although the general profile is not the same, the position of this ionic peak is actually independent of the preparation method. This characteristic feature has already been noted in a previous SAXS study of halato-telechelic polymers [48]. Moreover, the SAXS curve for the PIS2M1/11.0 sample shows a shoulder at small $s$ values in addition to the ionic peak, which might indicate a more ordered structure in this sample prepared by acid-base reaction rather than by ion-exchange. A previous SAXS study of similar samples $[29,41]$ showed oscillations in the SAXS curves measured with a Kratky camera. Although no explanation was provided, these oscillations might be related to a supramolecular organization of the multiplets. Oscillations and shoulders are observed in several samples considered in this study (Fig. 18).

That the proton-transfer is incomplete in mesogenic carboxylate containing samples is supported by the SAXS profiles of PIC1M1/4.5 and PIC1M2/4.5 samples. No ionic peak is indeed observed for the sample prepared by acid-base reaction in contrast to what happens when ion-exchange is carried out. The ionic and mesogenic peaks are no longer observed in the SAXS curves of poly(tert-butylacrylate) containing samples, whatever the method of preparation. A lack of contrast has already been noted in carboxylato poly(tert-butylacrylate) (Section 3), so that no clear conclusion can be drawn from the SAXS study of these compounds.

Association of the ion-pairs into multiplets is the expected supramolecular structure for $\mathrm{LCH}$ containing the M3 and M4 molecules, since these compounds do not form mesophases. In this respect, an ionic peak is observed for the PIS2M4/11.0, PIC1M4/4.5 and annealed PIS2M3/11.0, PIC1M3/4.5 
samples (Fig. 19), which is the signature of the dipolar association of the ion-pairs. The position of this ionic peak slightly differs for the M3 and M4 containing compounds compared to the M1 and M2 counterparts (Table 8). This observation suggests that the structure of the ionic cores is not the same for the M1 and M2 LC as for the non-LC M3 and M4 molecules.

The SAXS curves for PIS2M3/11.0 and PIS2M4/11.0 are identical (Fig. 19), which supports the completeness of the proton-transfer in the PIS2M3/11.0 sample. The same ionic peak is observed for the annealed PIC1M3/4.5 and PIC1M4/4.5 compounds (Fig. 19). Although no ionic peak is detected in PSC1M4/4.5 and annealed PSC1M3/4.5 (Fig. 19), these two samples show similar SAXS profiles, in agreement with a complete proton-transfer reaction in annealed PSC1M3/4.5. In sharp contrast to M1 containing samples, the proton-transfer reaction is effective when the more basic M3 molecule is used, in complete agreement with the DSC and FTIR data.

As observed in Fig. 18, some M3 and M4 containing samples in Fig. 19 (PSC1M3/4.5, PSC1M4/4.5, PSS 1M3/5.5, PIS2M3/11.0, etc.) show a shoulder in the small s region, which suggests a supramolecular organization of the multiplets.

Annealing is a prerequisite for the proton-transfer from the acid to the amine to be complete, whatever the acid, i.e. sulfonic or carboxylic. This is illustrated by the sharp difference in the SAXS curves for samples prepared by ion-exchange (PSS1M4/5.5, Fig. 20) and by acid-base reaction without annealing (PSS1M3/5.5, Fig. 20).

Table 8 Distance associated to the ionic peak $\left(d_{I P}\right)$ of M2 and M4 containing LCH

$$
\mathrm{d}_{\mathrm{IP}}(\mathrm{nm}), \mathrm{Z}=\mathrm{d}_{\mathrm{IP}}(\mathrm{nm}), \mathrm{Z}=
$$

M2 M4

PSS1Z/5.5 $7.4 \quad 8.1$

$\mathrm{PIC} 1 \mathrm{C} / 4.5 \quad 8.2 \quad 7.2$

PIS2Z/11.0 8.7

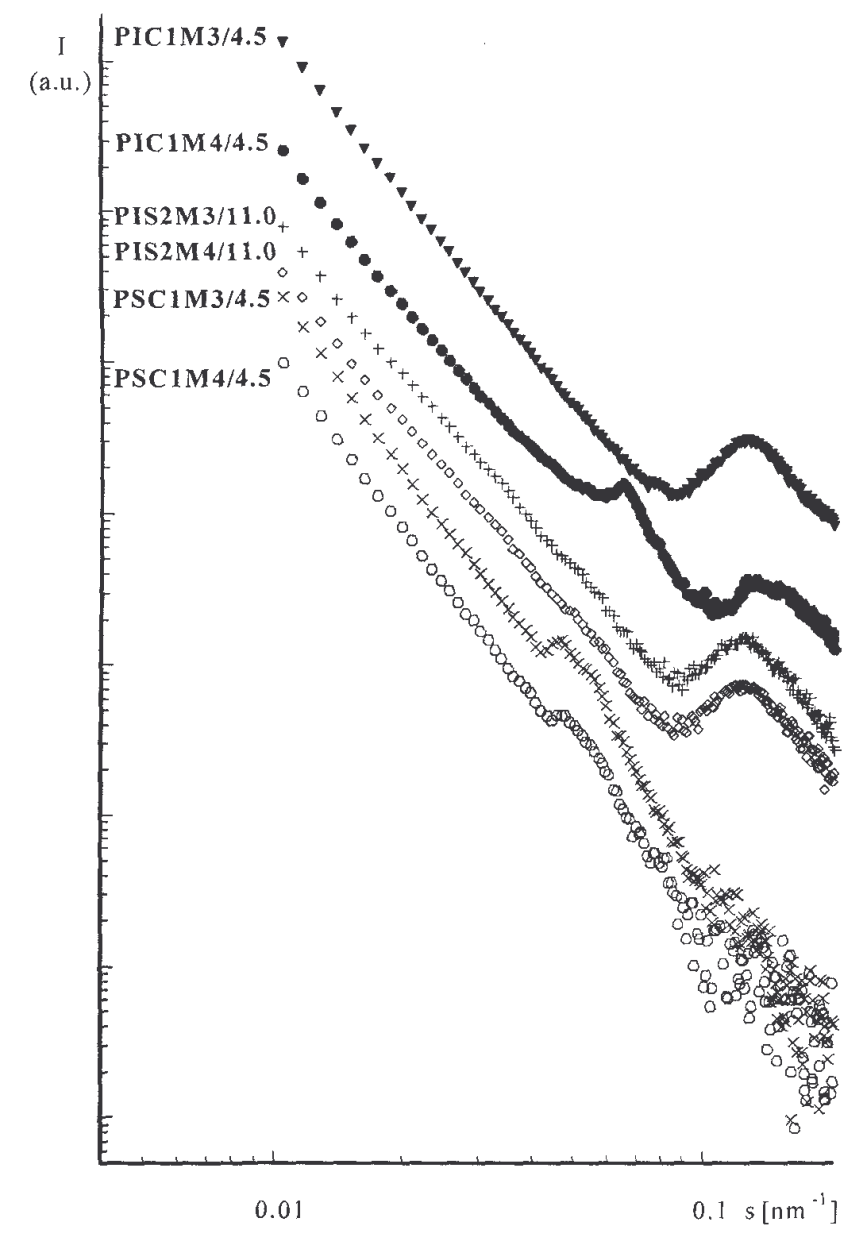

Fig. 19. SAXS curves for annealed M3 and M4 containing LCH. 
It must be noted that the shoulder in the small angle region is observed in the non-annealed PSS1M3/5.5 sample, although it is not detected for the parent compounds prepared by ion-exchange. The assignment of this shoulder to a supra-molecular structure is thus far from being clear. The effect of temperature on the SAXS curves was also examined. The SAXS profile for the annealed PSC1M3/4.5 sample clearly changes in the temperature range from 25 to $150^{\circ} \mathrm{C}$ (Fig. 21). The dramatic decrease in the intensity of the ionic peak can be related to the loss of the multiplet organization, as noted in Section 4.

In conclusion, SAXS data are in good agreement with the experimental observations by DSC, FTIR and POM.

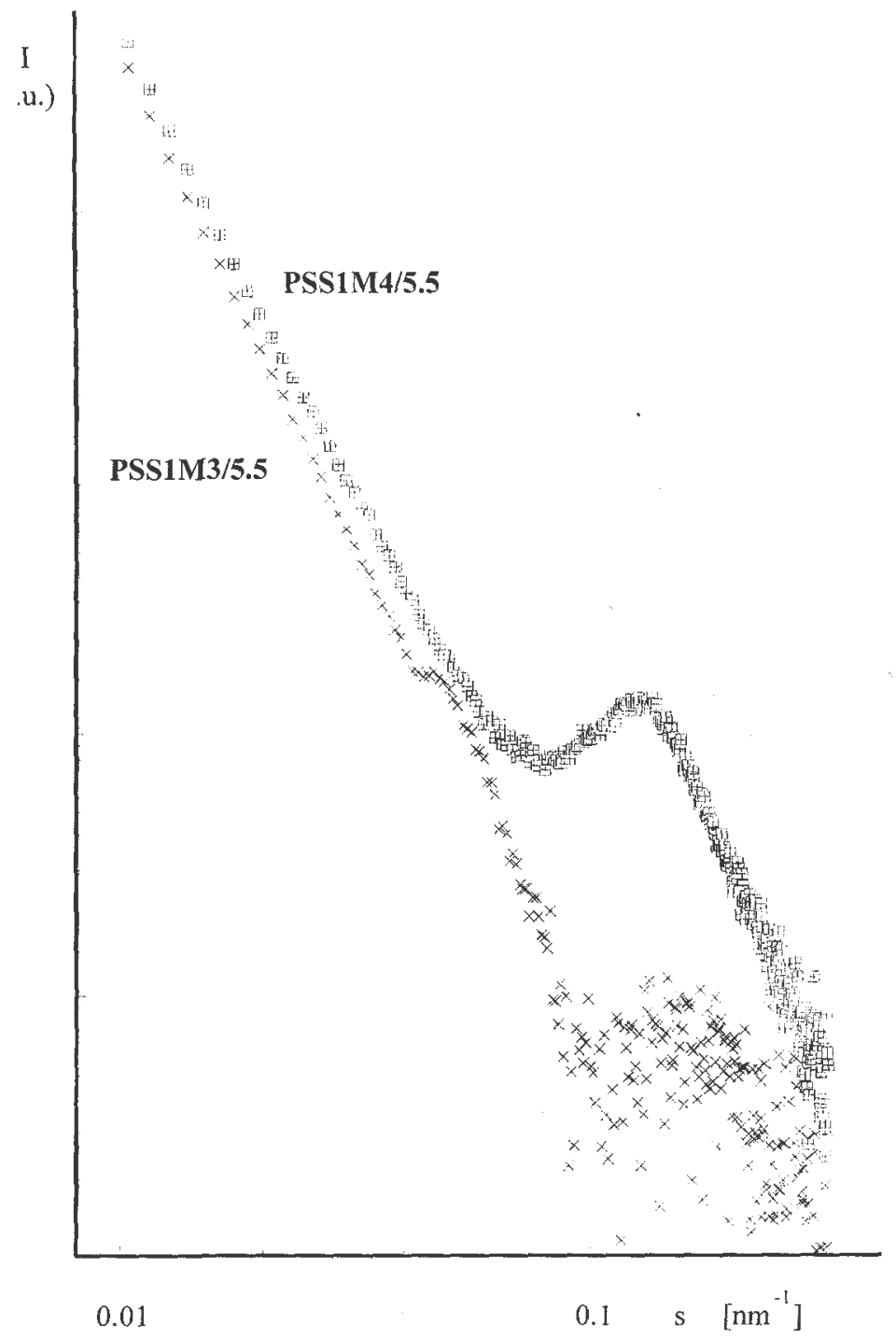

Fig. 20. SAXS curves for PSS1M4/5.5 and PSS1M3/5.5 before annealing.

\section{Use of LCH as interfacial agents in polymer dispersed liquid crystals}

Thin polymer films in which microphases of a low molecular weight liquid crystal (LC) are homogeneously dispersed have potential in a variety of electro-optical applications such as switchable windows [62] and active matrix projection displays [63]. They are commonly referred to as polymerdispersed liquid crystals (PDLC) [64]. Although blending of a conventional thermoplastic with an immiscible thermotropic LC is a straightforward way to PDLC, control of the final dispersion and ultimate properties may be quite a problem. In this respect, it is well known that phase dispersion and interfacial adhesion in immiscible polymer blends are dramatically improved by the use of block or graft copolymers, each constitutive component of which is selectively miscible with one phase of the polyblend [65]. 


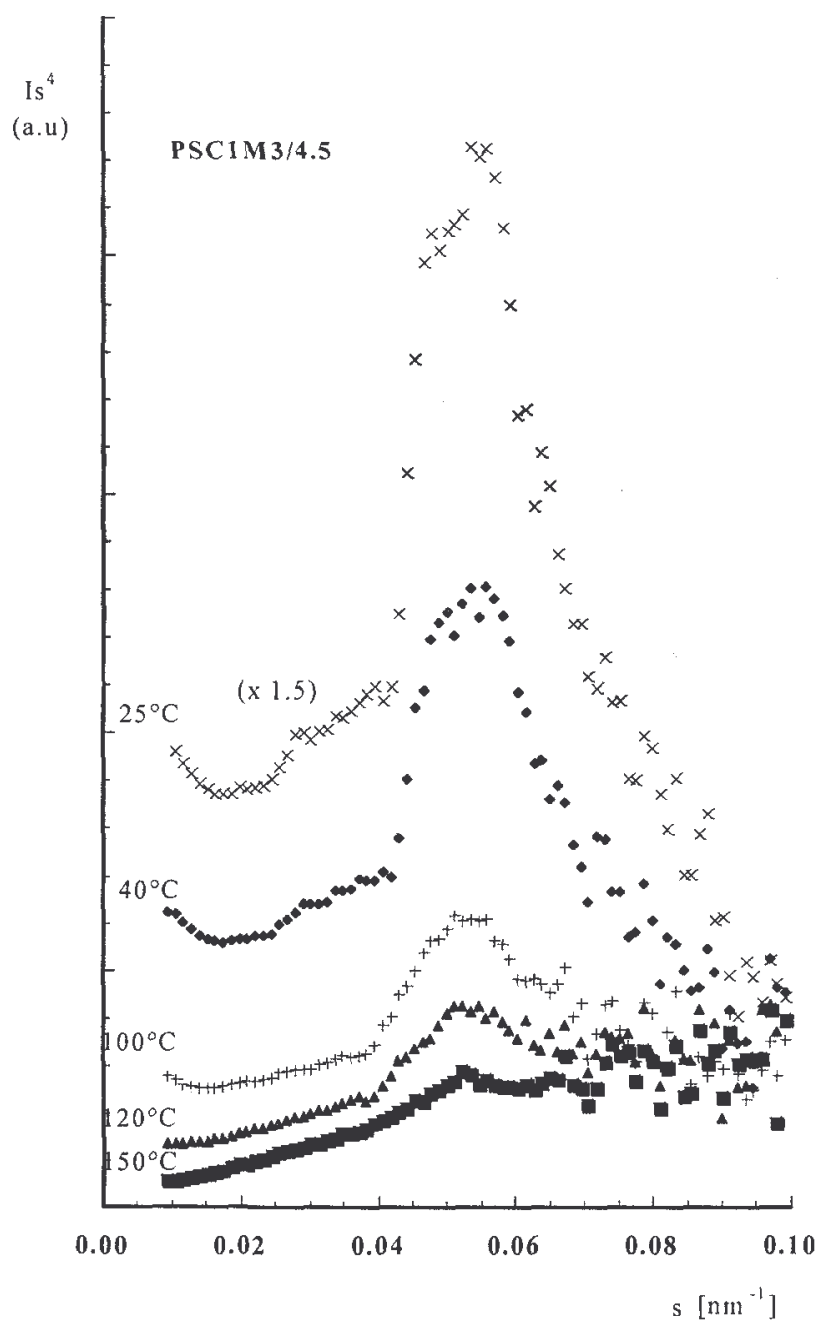

Fig. 21. Effect of temperature on the SAXS profile of the annealed PSC1M3/4.5 sample.

By analogy with that concept, an interfacial agent in PDLC might consist of a chain identical to the polymer matrix to which is bonded a component miscible or strongly interacting with the $\mathrm{LC}$ to be dispersed. For this two-component chain to be located at the polymer/LC droplets interface, two conditions have to be fulfilled. The constitutive components of the envisioned interfacial agents must be mutually repulsive and the balance of their preferential interactions with one phase of the PDLC must be such that this additive has no tendency to migrate and accumulate in either the polymer matrix or the LC. If it is so, size and shape of the dispersed microdroplets can be tailored in agreement with good electro-optical properties [66]. The bonding of the component miscible with the LC phase to the polymer chain may be either ionic or covalent. Since this characteristic feature may affect the balance of the interactions in the ternary system, the comparison of the interfacial activity of the ionic and covalent structures in PDLC is highly desirable. 
Table 9 Structure of the interfacial agent used in PDLC (covalent bonding of the mesogen to the polymer)
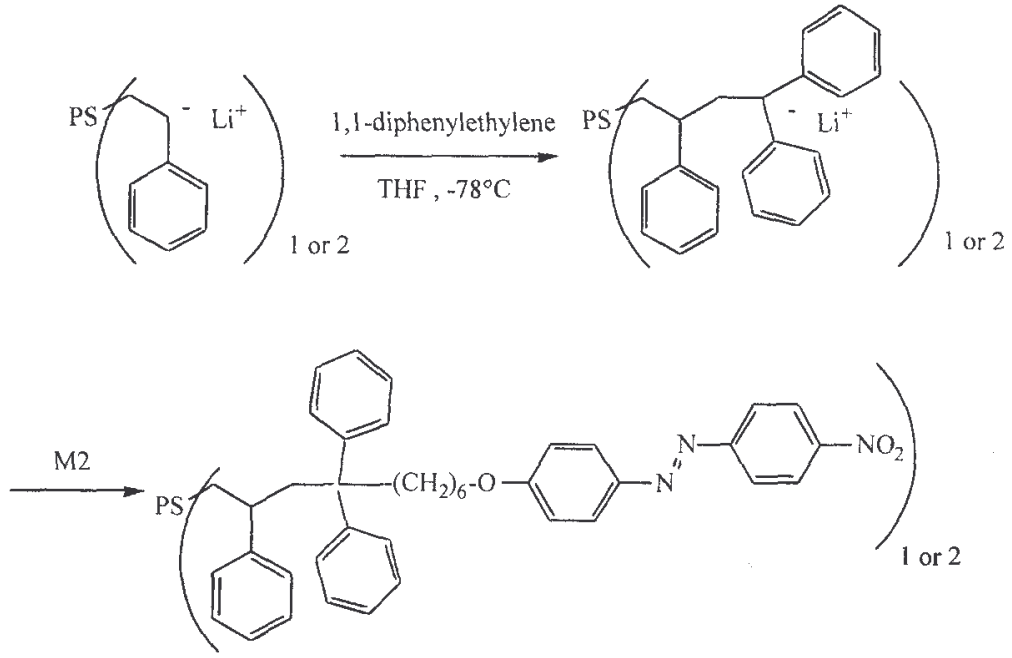

$\begin{array}{lll}\text { Sample } & M_{n} \text { polystyrene } & \text { Number of end-groups per chain } \\ \text { PS1M/4.0 } & 4000 & 1 \\ \text { PS2M/5.0 } & 5000 & 2\end{array}$

The amount of an ionic compound in PDLCs must be kept as small as possible so as to restrict conductivity in inherently electrical devices. This requirement will be met as easily as the interfacial activity of this compound will be high.

Several interfacial agents were selected that contain a polystyrene chain capped by a mesogenic component through a covalent bond and an ionic bond, respectively. These two types of interfacial agents were compared in a dispersion of a LC in an immiscible polystyrene matrix. The low molar mass M1 liquid crystal was selected to be dispersed as droplets in polystyrene.

The first type of interfacial agent, shown in Table 9, is a low molecular weight (semi)telechelic polystyrene chain ((S)TP) covalently capped at (one or) both end(s) by a mesogenic moiety. The synthesis and thermal properties of these compounds were reported elsewhere [28]. Ionic interfacial agents were LCH containing a polystyrene backbone.

The mesogen M1 is immiscible with the non-functional polystyrene as supported by the DSC trace of a blend of this mesogen (50\% weight) and polystyrene $\left(\bar{M}_{n}=58,000\right)$, which is the superposition of the traces of each component. Optical microscopy confirms the immiscibility of M1 and polystyrene since randomly dispersed liquid crystalline domains are clearly seen in the polymer matrix (Fig. 22a). Miscibility can however be observed by heating the M1/polystyrene blend above the isotropization temperature of $\mathrm{M} 1\left(120^{\circ} \mathrm{C}\right) .5 \mathrm{wt} \%$ of each type of interfacial agents was added to a $50 / 50$ polystyrene/ mesogen M1 blend, dissolved in chloroform. Chloroform-cast films were observed by optical microscopy under crossed polarizers, in order to compare the efficiency of the interfacial agents.

Fig. 22b shows the film obtained when the PS1M/4.0 interfacial agent is added. This film is essentially the same as the film shown in Fig. 22a, indicating that the non-ionic interfacial agent PS1M/4.0 has no influence on the LC dispersion. 


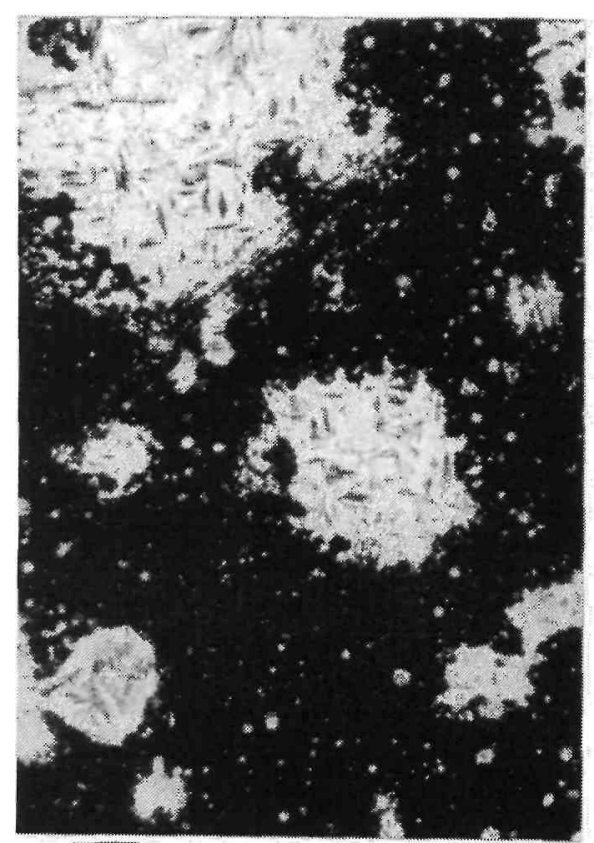

$25 \mu$

a

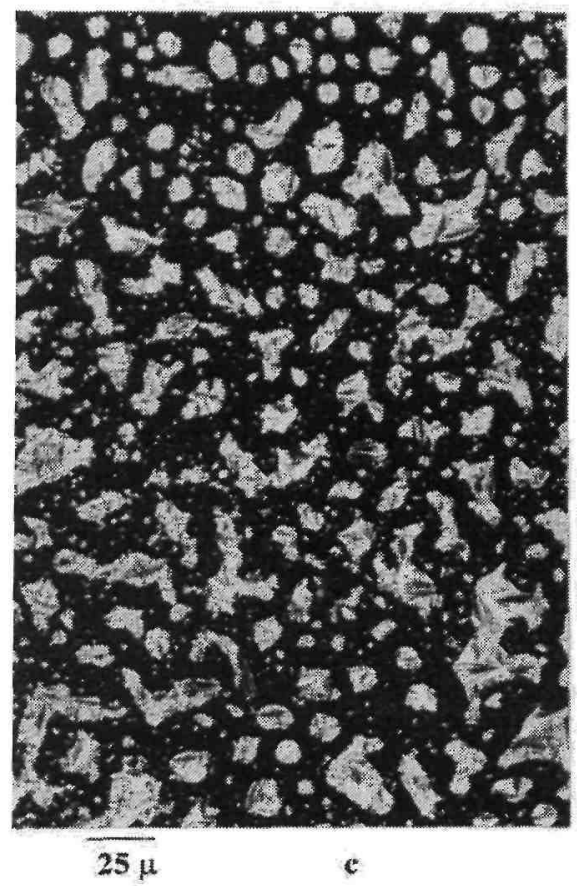

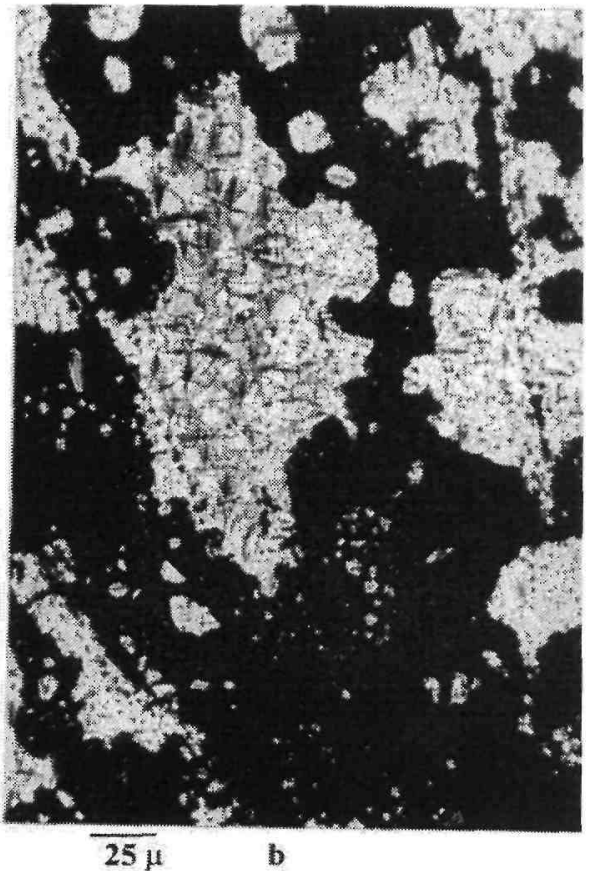

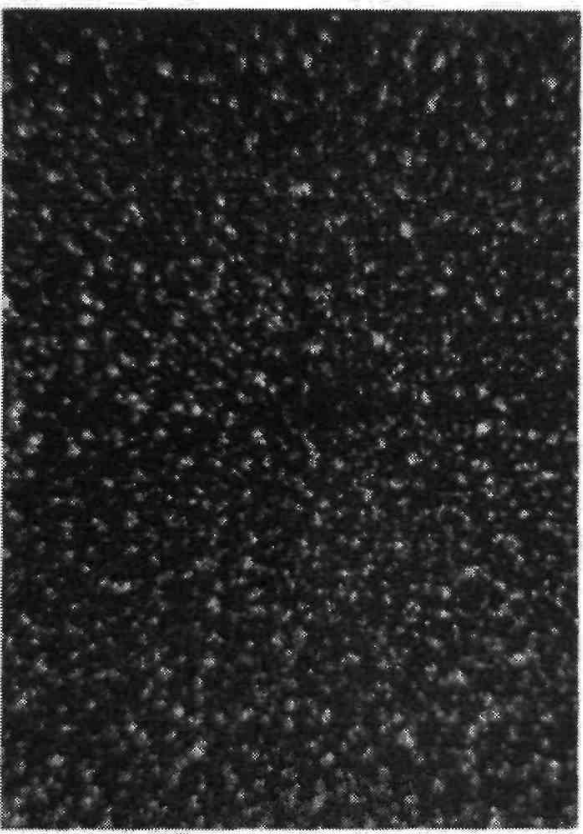

$25 \mu$

d

Fig. 22. Droplets of mesogen M1 dispersed in a polystyrene matrix without (a), with (b) the PS1M/4.0 interfacial agent (covalent bonding), with (c) carboxylate containing interfacial agent (PSC1M2/6.0) and with (d) sulfonate containing inter-facial agent (PSS1M2/5.5). (Observation temperature $=20^{\circ} \mathrm{C}$, crossed polarizers, magnification $=400 x$ ).

The PS2M/5.0 of a higher molar mass leads to the same observation. Optical microscopy of chloroform-cast films shows that the non-ionic interfacial agents, PS1M/4.0 and PS2M/5.0, are miscible to polystyrene in the whole composition range. Furthermore, these (S)TPs are poorly miscible with the mesogen M1, which is already apparent in a 10/90 (S)TPs/M1 blend. Therefore (S)TPs are poor interfacial agents for polystyrene/M1 blends because of a too high solubility in polystyrene and lack of migration to the LC/polymer interface. When the PSC1M2/6.0 ionic interfacial agent is added to the polystyrene/M1 blend, droplets $(10-20 \mu \mathrm{m})$ are homogeneously dispersed throughout the polystyrene matrix (Fig. 22c). Fan-shaped textures are observed within the droplets which are typical of smectic mesophases. The same morphology is observed when the difunctional PSC2M2/6.0 
interfacial agent is added.

Spherical micrometer-sized mesogen droplets are formed when a sulfonate $\mathrm{LCH}$ ionic interfacial agent is used. Fig. 22d illustrates this interesting situation for the PSS1M2/5.5 sample. Morphology of this film is quite suitable to application in the PDLC field. The size of the droplets is slightly reduced from ca. 1 to $0.5 \mu \mathrm{m}$ when $2.5 \mathrm{wt} \%$ of the difunctional PSS2M2/5.5 is added instead of the monofunc-tional counterpart $(5 \mathrm{wt} \%)$. Thus, at the same sulfonate end-groups content, difunctional sulfonato $\mathrm{LCH}$ are more efficient than monofunctional ones in reducing the interfacial tension and/or preventing coalescence of the dispersed LC phases. This effect could be related to loop formation by the difunctional sulfonate $\mathrm{LCH}$ at the $\mathrm{LC} /$ polymer interface. This conformation was previously observed when $\alpha$ and $\alpha, \omega$-sulfonato polystyrene chains adsorbed on silicon wafers [67]. It was shown indeed that the effective grafting density of the difunctional polystyrene chains in the first layer is lower than that of the monofunctional chains, due to loop formation.

$\mathrm{LCH}$ act as interfacial agents at the polystyrene/M1 interface. This interfacial activity has to be attributed to the presence of the ion pairs which are able to migrate at the interface. Sulfonate LCH are more efficient that carboxylato ones as supported by a finer mesogen dispersion. The reason for this observation should be found in the structure and polarity of the sulfonates and carboxylates ion-pairs, respectively. The more polar sulfonate $\mathrm{LCH}$ are less soluble in both the polystyrene and the LC phases than the carboxylato ones, and they are thus preferentially localized at the polystyrene/LC interface, which explains their interfacial activity. Thus, when a small organic molecule is covalently bonded to a polymer chain as an end-group, the mutual repulsion between these two constitutive components is not strong enough to prevent the final macromolecule from accumulating in one phase of the parent organic molecule/polymer blend. The balance of interactions is however deeply modified when these two constituents components are bonded to each other by an ionic linkage, to the point where the additive is no longer localized in one phase of the blend but rather at the interface.

\section{Conclusions}

The mesogenic properties of novel liquid crystalline halato(semi)telechelic polymers have been studied by a set of experimental techniques, i.e. DSC, POM and SAXS. Liquid crystalline mesophases can be formed in $\mathrm{LCH}$, although the weight percentage of intrinsically $\mathrm{LC}$ moieties (in the range of ca. $5-40 \%$ in $\mathrm{LCH}$ ) is considerably lower than in classical LC side-chain or main-chain polymers (generally of the order of 50-80\%). This interesting conclusion is thought to result from the microphase separation between the mesogenic cations and the polymer is $\mathrm{LCH}$, with formation of two possible supramolecular structures. The first one shows as isotropic texture and an ionic peak in the SAXS pattern, which is the signature of ionic microdomains. From the position of the ionic peak, an average distance between the nearest neighbor microdomains was calculated, that however changes with the polymer molecular weight and chemical nature. Indeed, the position of the ionic peak changes as the square root of the molecular weight, provided that the experimental correlation length is corrected for a restricted chain mobility region, whose the thickness is the persistence length of polystyrene, and for the thickness of the mesogenic cations. These observations are in agreement with a rod-like organization of the multiplets. A texture characteristic of a smectic A mesophase is typical of the second supramolecular structure. A SAXS peak is observed, whose the position is dictated by the layer spacing in the mesophase.

So, it appears that the final morphology results from the competition between the dipolar interaction of the ion-pairs and the propensity of the mesogenic moieties to form mesophases. Depending on the strength of the mutual dipolar interactions, the flexibility of the polymer backbone, the mesogen/polymer weight ratio and the temperature, one of the two supramolecular structures dominates the phase morphology or both of them coexist. As a rule, the smectic mesophase is preferably formed when the mesogen/polymer weight ratio is high, the temperature is increased (although not higher than $T_{\mathcal{C}}$ ), the flexibility of the polymer backbone is high (PI rather than PS) and when the dipolar interactions are weak enough. In this respect, sulfonato end-groups have a detrimental effect on the mesophase formation.

Halato(semi)telechelic polymers were end-capped by mesogens by either ion-exchange or protontransfer reaction. The effectiveness of the proton-transfer strongly depends on the acid/base pair involved. In this respect, sulfonic acid-ended polymers are ideal candidates for reaction with aminated mesogens. The situation is quite different when carboxylic acids are concerned. Two cases have to be considered, depending on the basicity of the aminated M1 and M3 molecules.

Hydrogen-bonds with strong polarization of the hydrogen atom are detected by FTIR when M1 is mixed with carboxylic acid-ended polymers. A partial proton transfer is however observed after annealing. In sharp contrast, a nearly complete proton-transfer is observed when the more basic M3 
molecule is used. However, the proton-transfer reaction needs annealing to be complete. Finally, it appears that LC halato(semi)telechelic polystyrenes act as interfacial agents at the polystyrene/M1 interface. This interfacial activity is promoted by the ion pairs which are able to migrate at the interface. Sulfonato LCH are more efficient that the carboxylato ones as supported by the finer mesogen dispersion.

\section{Acknowledgements}

JFG and RJ are very much indebted to the Services Fédéraux des Affaires Scientifiques, Techniques et Culturelles for financial support in the frame of the Poles d'attraction Interuniversitaires: 4-11: Chimie Supramoleculaire et Catalyse Supramoleculaire. JFG greatly acknowledges the SUPERNET and the Training and Mobility of Researchers Programme of the European Community for financial support to the SAXS experiments at L.U.R.E. (Orsay, France) and to the SANS measurements at the Laboratoire Leon Brillouin (Saclay, France).

\section{References}

[1] Shibaev VP, Plate NA. Liquid crystal polymers, Advanced Polymer Science 60/61. Berlin: Springer, 1984.

[2] Paleos C, Filippakis S, Margomenou-Leonidopoulou G. J Polym Sci, Polym Chem Ed 1981; 19:1427.

[3] Imrie CT. Trends Polym Sci 1995;3:22.

[4] Lehn JM. Angew Chem Int Ed Engl 1990;29:1304.

[5] Kato T, Frechet JM. Macromolecules 1989;22:3819.

[6] Kumar U, Kato T, Frechet JMJ. J Am Chem Soc 1992;114:6630.

[7] Kato T, Kihara H, Uryu T, Frechet JMJ. Angew Chem Int Ed Engl 1994;33:1644.

[8] Kumar U, Frechet JMJ, Kato T, Ujiie S, Iimura K. Angew Chem Int Ed Engl 1992;31:1531.

[9] Kato T, Nakano M, Moteki T, Uryu T, Ujiie S. Macromolecules 1995;28:8875.

[10] Kato T, Kihara H, Uryu T, Fujishima A, Frechet JMJ. Macromolecules 1992;25:6836.

[11] Malik S, Dhal PK, Mashelkar RA. Macromolecules 1995;28:2159.

[12] Fouquey C, Lehn JM, Levelut AM. Adv Mater 1990;2:254.

[13] Bladon P, Griffin AC. Macromolecules 1993;26:6604.

[14] Lee CM, Jariwala CP, Griffin AC. Polymer 1994;35:4550.

[15] Ujiie S, Iimura K. Macromolecules 1992;25:3174.

[16] Ujiie S, Iimura K. Polym J 1993;25:347.

[17] Ujiie S, Iimura K. Chem Lett 1991:411.

[18] Ujiie S, Iimura K. Chem Lett 1990:995.

[19] Bazuin CG, Tork A. Macromolecules 1995;28:8877.

[20] Ujiie S, Tanaka Y, Iimura K. Chem Lett 1991:1037.

[21] Masson P, Gramain P, Guillon D. Macromol Chem Phys 1995;196:3677.

[22] Masson P, Heinrich B. Macromol Chem Phys 1994;195:1199.

[23] Navarro-Rodriguez D, Frere Y, Gramain P. Macromol Chem Phys 1991; 192:2975.

[24] Navarro-Rodriguez D, Guillon D, Skoulios A, Frere Y, Gramain P. Macromol Chem Phys 1992;193:3177.

[25] Zhao Y, Huanli L. Macromolecules 1994;27:4525.

[26] Zhang B, Weiss RA. J Polym Sci, Polym Chem Ed 1992;30:91.

[27] Gohy JF, Vanhoorne P, Jérôme R. Macromolecules 1996;29:3376.

[28] Gohy JF, Jerome R. Macromol Chem Phys 1996; 197:2209.

[29] Sobry R, Fontaine F, Van den Bossche G, Gohy JF, Jérôme R. J Appl Crystallogr 1997;30:1075. [30] Gohy JF, Van den Bossche G, Sobry R, Jérôme R. Macromol Chem Phys 1998; 199:1791.

[31] Joachimi D, Lattermann G, Schellhorn M, Tschierke C, Zugenmaier P. Liq Cryst 1995;18:303. [32] Register RA, Cooper SL, Thyagarajan P, Chakrapani S, Jerome R. Macromolecules 1990;23:2978. [33] Broze G, Jerome R, Teyssié P. Macromolecules 1982; 15:920.

[34] Quirk RP, Kim J. Macromolecules 1991;24:4515.

[35] Fayt R, Forte R, Jacobs C, Jérôme R, Ouhadi T, Teyssié P, Varshney SK. Macromolecules 1987;20:1442.

[36] Gray GW, Goodby JW, editors. Smectic liquid crystals. Glasgow, London: Leonard Hill, 1984. [37] Demus D, Richter L, editors. Textures of liquid crystals Weinheim: Verlag Chemie, 1978.

[38] Bazuin CG, Guillon D, Skoulios A, Nicoud JF. Liq Cryst 1986; 1:181

[39J Coles HJ, Simon R. Polymer 1985;26:1801.

[40] Charlier P, Jérôme R, Teyssié Ph, Prud'homme RE. J Polym Sci, Polym Chem Ed 1993;31:129. [41] Fontaine F, Ledent J, Sobry R, Francois E, Jérôme R, Teyssié P. Macromolecules 1993;26:1480. [42] Horrion J, Jérôme R, Teyssié P, Marco C, Williams CE. Polymer 1988;29:1203.

[43] Williams CE, Russel T, Jérôme R, Horrion J. Macromolecules 1986;19:1203.

[44] Vlaic G, Williams CE, Jérôme R, Tant MR, Wilkes GL. Polymer 1987;29:173.

[45] Pineri M, Eisenberg A. Structure and properties of ionomers, NATO ASI series C 198. Boston: D. Reidel, 1987.

[46] Eisenberg A, Hird B, Moore RB. Macromolecules 1990;23:4098.

[47] Higgins JS, Benoit HC. Polymers and neutron scattering. Oxford: Clarendon Press, 1994.

[48] Vanhoorne P, Van den Bossche G, Fontaine F, Sobry R, Jerome R, Stamm M. Macromolecules 1994;27:838.

[49] Lundberg RD, Makowski HS. In: Eisenberg A, editor. Ions in polymers, Advances in chemistry series 187. Washington, DC: ACS, 1980.

[50] Righdal M, Eisenberg A. J Polym Sci, Polym Phys Ed 1981;19:287.

[51] Lefelar JA, Weiss RA. Macromolecules 1984;17:1145. 
[52] Wyckoff RWG. Crystal structures. 2nd ed. New York: Interscience, 1969.

[53] Vanhoorne P, Jérôme R, Teyssié P, Lauprêtre F. Macromolecules 1994;27:2548.

[54] Gao Z, Xing-Fu Z, Eisenberg A. Macromolecules 1994:27:794.

[55] Yano S, Tadano K, Jérôme R. Macromolecules 1991;24:6439.

[56] Tsagaropoulos G, Kim J-S, Eisenberg A. Macromolecules 1996;29:2222.

[57] Young RJ, Lovell PA. Introduction to polymers. London: Chapman \& Hall, 1991.

[58] Bazuin CG, Brandys FA. Chem Mater 1992;4:970.

[59] Bazuin CG, Brandys FA, Eve TM, Plante M. Macromol Chem, Macromol Symp 1994;84:183.

[60] Lebedeva TL, Shandryuk GA, Sycheva TI, Bezborodov VS, Tal'roze RV, Plate NA. J Mol Struct 1995;354:89.

[61] Tal'roze RV, Kupstov SA, Sycheva TI, Bezborodov VS, Plate NA. Macromolecules 1995;28:8689.

[62] Doane JW. In: Bahadur B, editor. Liquid crystals — applications and uses, vol. 1. Singapore: World Scientific, 1990.

[63] Montgomery GP. Proc SPIE I 1989;54:577.

[64] Doane JW, Golemme A, West JL, Whitehead JB, Wu BG. Mol Cryst Liq Cryst 1988;165:511.

[65] Fayt R, Jérôme R, Teyssié Ph. Macromol Chem 1986;187:837.

[66] Drzaic PS. Proc SPIE 1989;1080:11.

[67] Pankewitsch T, Vanhoorne P, Jerome R, Stamm M. Macromolecules 1995;28:6986. 